\title{
The twisted Drinfeld double of a finite group via gerbes and finite groupoids
}

\author{
SimON WiLlERTON
}

\begin{abstract}
The twisted Drinfeld double (or quasi-quantum double) of a finite group with a 3cocycle is identified with a certain twisted groupoid algebra. The groupoid is the loop (or inertia) groupoid of the original group and the twisting is shown geometrically to be the loop transgression of the 3-cocycle. The twisted representation theory of finite groupoids is developed and used to derive properties of the Drinfeld double, such as representations being classified by their characters.

This is all motivated by gerbes and 3-dimensional quantum field theory. In particular the representation category of the twisted Drinfeld double is viewed as the "space of sections" associated to a transgressed gerbe over the loop groupoid.
\end{abstract}

57R56; 16W30, 18B40

\section{Introduction}

There were several motivations for this work. The first was to understand what the twisted Drinfeld double of a group really is, and in particular to understand exactly what the twisting is. The second was to see how well Dijkgraaf-Witten quantum field theory worked in the two-gerbe paradigm. The third was to understand the FreedHopkins-Teleman result on twisted equivariant K-theory in this baby example. From this one might then hope to gain some insight into Chern-Simons theory.

The twisted Drinfeld double of a finite group arose in the work of Dijkgraaf, Pasquier and Roche in relation to orbifold conformal field theory and was shown by Freed [8] (following Freed and Quinn [9] and Altschuler and Coste [1] to be the relevant quantum group for the quantum field theory from a finite group twisted by a cocycle. Given a finite group $G$ and a three-cocycle $\omega \in Z_{\mathrm{gp}}^{3}(G, U(1))$ the twisted Drinfeld double $D^{\omega}(G)$ can be defined to be the algebra over the complex numbers, with a basis $\{\langle\stackrel{g}{\leftarrow} x\rangle \mid g, x \in G\}$ and with the product defined by

$$
\langle\stackrel{h}{\leftarrow} y\rangle \cdot\langle\stackrel{g}{\leftarrow} x\rangle:=\delta_{y, g x g^{-1}} \frac{\omega(h, g, x) \omega\left(h g x(h g)^{-1}, h, g\right)}{\omega\left(h, g x g^{-1}, g\right)}\langle\stackrel{h g}{\leftarrow} x\rangle .
$$


Freed showed that in the untwisted case this can be thought of as the groupoid algebra of a certain finite groupoid. In this paper I develop that idea considerably further and show that the scalar factor in the definition of the product - the twisting - is essentially a two-cocycle $\tau(\omega)$ on the loop (or inertia) groupoid $\Lambda \bar{G}$, and that the twisted Drinfeld double is the corresponding twisted groupoid algebra.

Following that, I then show that many properties of the twisted Drinfeld double-such as its semisimplicity, its character theory and the decomposition of its representation group-follow from the fact that it is a twisted groupoid algebra. (Some of these properties have been algebraically presented in the somewhat terse paper of Altschuler, Coste and Maillard [2].) The twisted representation theory of finite groupoids, which is developed here, is hardly more complicated than the twisted (or projective) representation theory of finite groups which, in turn, is hardly more complicated than the ordinary representation theory of finite groups. In fact looking at the twisted representation theory of finite groupoids throws some more light on the twisted representation theory of finite groups, so that the notion of twisted characters is just an example of the transgression phenomenon.

From the point of view of gerbes the key topological construction is the Parmesan map. I show how the $S^{1}$-transgression map is expressed in general for $n$-gerbes on finite groupoids. To be more specific, by an $n$-gerbe on a finite groupoid I really mean a $U(1)$-valued $(n+1)$-cocycle on $\mathcal{G}$. Associated to $\mathcal{G}$ is the loop groupoid (or the inertia groupoid) and the transgression map associates an $n$-cocycle on the loop groupoid. This is the Parmesan construction of Section 1 and it is that which allows us to move up and down the gerbe ladder.

It should be pointed out here that as it is just finite groupoids being considered, the terms "loop groupoid" and "inertia groupoid" can be used interchangeably. In more general situations this is not the case; more comments on this are made at the end of this introduction.

From the point of view of quantum field theory we see how Dijkgraaf-Witten theory fits into the two-gerbe philosophy. To explain this it is useful to have a digression on $n$-gerbes and transgression.

\section{TQFTs and $n$-gerbes}

Although not strictly necessary for understanding the results of this paper, the following will give some idea of the thinking behind them. 
Topological quantum field theory Suppose $G$ is a compact group. If $G$ is finite then we will be considering Dijkgraaf-Witten theory; if $G$ is simply connected and simple then we will be considering Chern-Simons theory. Given a "level" $k \in H^{4}(B G, \mathbb{Z})$ we can construct a three-dimensional topological quantum field theory. This means, vaguely, to each closed three-manifold we associate a complex number, to each closed two-manifold we associate a vector space and to each three manifold with boundary we associate a vector in the vector space associated to its boundary. In fact we might want to go further and to each closed one-manifold associate a category and even associate to each closed zero-manifold a two-category. The physical way to do this (at least at the vector space and vector level) is using path integrals; so for instance the invariant of a closed three-manifold $M$ is the integral of a certain action over the space of connections on a $G$ bundle over $M$, and the vector space associated to a two-manifold, $\Sigma$ is a certain space of sections of a line bundle over the "moduli space" of flat $G$-connections on $\Sigma$. The philosophy of the current paper, which I learnt from Freed (see for example [8]) and Brylinski and McLaughlin [5], is that the hierarchy of $n$-gerbes gives a mathematical framework for trying to understand these path integrals.

Transgression and $n$-gerbes Implicit in Dijkgraaf and Witten's work is that it is sensible to interpret the level $k \in H^{4}(B G, \mathbb{Z})$ as a two-gerbe with connection. Being slightly vague, a two-gerbe is a geometric representative of a class in $H^{4}(X, \mathbb{Z})$, just as a principal $U(1)$-bundle is a geometric representative of a class in $H^{2}(X, \mathbb{Z})$. There are many ways of representing a class in a "geometric" way, but for the sake of this discussion, we can take a two-gerbe to be a sort of bundle of two-categories. There is a hierarchy of $n$-gerbes, which we can indicate as follows:

\begin{tabular}{c|c|c}
$n$ & "n-gerbe" & represents a class in... \\
\hline-2 & $\mathbb{Z}$-valued function & $H^{0}(X, \mathbb{Z})$ \\
-1 & $U(1)$-valued function & $H^{1}(X, \mathbb{Z})$ \\
0 & principal $U(1)$-bundle & $H^{2}(X, \mathbb{Z})$ \\
1 & "bundle of categories" & $H^{3}(X, \mathbb{Z})$ \\
2 & "bundle of two-categories" & $H^{4}(X, \mathbb{Z})$
\end{tabular}

In the geometric ways of representing higher gerbes there are appropriate notions of connections, generalizing that on $U(1)$-bundles, and an $n$-gerbe with connection, essentially by definition, represents a class in the more subtle Deligne cohomology group. For $n$-gerbes with connection there are notions of holonomy and transgression, the basic example of which is got from a $U(1)$-bundle-with-connection on a space $X$. The holonomy of this can be thought of as a $U(1)$-valued function on the loop space of $X$, so there is a way of going from a 0 -gerbe (with connection) on $X$ to a 
$(-1)$-gerbe (with connection) on $\operatorname{Maps}\left(S^{1}, X\right)$. Similarly given a one-gerbe on $X$, this can be transgressed to a $U(1)$-bundle with connection on the loop space or to a $U(1)$-valued function on the space of maps of a surface $\Sigma$ into $X$. More generally, for an oriented, closed $m$-manifold $Y$, with $0 \leq m \leq n+2$, there is a transgression map

$\{n$-gerbes with connection on $X\}$

$\longrightarrow\{(n-m)$-gerbes with connection on $\operatorname{Maps}(Y, X)\}$,

which on the level of cohomology is just the composition

$$
H^{n+2}(X, \mathbb{Z}) \stackrel{\mathrm{ev}^{*}}{\longrightarrow} H^{n+2}(\operatorname{Maps}(Y, X) \times Y, \mathbb{Z}) \stackrel{\int_{Y}}{\longrightarrow} H^{(n-m)+2}(\operatorname{Maps}(Y, X), \mathbb{Z}),
$$

where ev: Maps $(Y, X) \times Y \rightarrow X$ is just the evaluation map. This is constructed on the level of Deligne cohomology by Gomi and Terashima [11].

It its possible to make sense of transgression for $m$-manifolds with boundary and that is essential in setting up the full quantum field theory but it is not something that we will be using here. The next thing to do is to consider the "spaces of sections".

Spaces of sections of gerbes In order to build a topological quantum field theory we need to understand the "space of sections" of an $n$-gerbe, which is a higher analogue of geometric quantization. The term "space of sections" is not entirely adequate, and perhaps "integral" or "pushforward to a point" would be better. First consider the case of a $U(1)$-bundle: to obtain its space of sections we must tensor with the complex line to obtain a line bundle and then we take the sections of that to obtain a vector space. What we really want at this point is a finite-dimensional vector space and this is where we get to the nub of geometric quantization, the thorny issue of polarization. Finding a polarization results in cutting down drastically the number of sections that we consider, for instance if a holomorphic structure exists on the base and the bundle, then we can restrict to holomorphic sections, obtaining a finite dimensional space of section in the case of a compact base. Ignoring the issue of polarization for a moment, the correct notion of space of sections for a $U(1)$-valued function is just the integral of the function, so that is a complex number. In the case of a gerbe, the "space of sections" (after the analogue of tensoring with the complex numbers) forms a linear category. The process of polarization for gerbes seems even more mysterious. Fortunately polarization is not an issue that has to be addressed in this paper as the base spaces are all discrete. So we can make sense of the space of sections of a gerbe.

Modulo the difficulties of defining the spaces of polarized sections, given an $n$-gerbe over a space $X$ we thus have a philosophy of obtaining a linear $(n-m)$-category from a closed, oriented $m$-manifold $Y$, namely take the space of (polarized) sections of the complexified gadget associated to the transgressed $(n-m)$-gerbe over $\operatorname{Maps}(Y, X)$. 
Stacks, groupoids and gerbes I actually want to work with "stacks" rather than spaces. This means that I want to take extra symmetry information into account and means in practice dealing with groupoids rather than spaces. So the points in the space are now objects of a groupoid and there is the extra information of the morphism sets between points. The example here is the stack of $G$-bundles on a compact manifold $M$, where $G$ is a finite group. (As $G$ is finite, these will necessarily be flat bundles.) To get a groupoid representing this stack we can take a finite-pointed model $\Pi_{1}(M)$ for the fundamental groupoid, by which I mean a groupoid whose objects correspond to a finite number of points on $M$, with at least one point on each connected component of $M$ and whose morphisms are the homotopy classes of paths between these points; in particular if $M$ is connected and $m_{0}$ is a base-point then we could take the groupoid with one object and with the fundamental group $\pi_{1}\left(M, m_{0}\right)$ as the group of morphisms. It is convenient to allow more than one point on each component, as this makes things more natural if we are considering spaces with boundaries. Now a groupoid representing the stack of flat $G$-bundles on $M$ is given by the groupoid of functors $\mathfrak{G}(M):=\mathcal{F} \operatorname{un}\left(\Pi_{1}(M), \bar{G}\right)$, where $\bar{G}$ is the groupoid with one object and $G$ as its group of morphisms, while the functor groupoid has functors as its object and natural transformations as its morphisms. Note two things here: firstly $\mathfrak{G}(M)$ is a finite groupoid as $\Pi_{1}(M)$ is finitely generated and $\bar{G}$ is finite. Secondly, $\Pi_{1}(M)$ is only defined up to equivalence, and hence so is $\mathfrak{G}(M)$.

To be concrete, here an $n$-gerbe on a finite groupoid will mean a $U(1)$-valued, $(n+1)-$ cocycle on the groupoid. We can define transgression similar to above, so that in particular if $\omega$ is a 3-cocycle on $G$ then for $M$ a closed (triangulated) $m$-manifold there is the transgression $\tau_{M} \omega$ which is a $(2-m)$-gerbe on $\mathfrak{G}(M)$. (The cocycle will actually depend on the triangulation of $M$, but its cohomology class will not.) We can associate a "complexified bundle" $\left(\tau_{M} \omega\right)_{\mathbb{C}}$ and take its space of sections to get a $(2-m)$-category $V(M):=\Gamma_{\mathfrak{G}(M)}\left(\left(\tau_{M} \omega\right)_{\mathbb{C}}\right)$ which is the invariant of $M$.

Thus given $G$ and $\omega \in Z_{\mathrm{gp}}^{3}(G, U(1))$ we get the sequence of assignments:

$$
\begin{aligned}
& \text { manifold: } \rightsquigarrow(2-m) \text {-gerbe over finite groupoid: } \rightsquigarrow \quad \text { space of sections: } \\
& M^{m} \quad \tau_{M} \omega \text { over } \mathfrak{G}(M)=\mathcal{F} \text { un }\left(\Pi_{1}(M), \bar{G}\right) \quad V(M):=\Gamma_{\mathfrak{G}(M)}\left(\left(\tau_{M} \omega\right)_{\mathbb{C}}\right)
\end{aligned}
$$

Dimension and $S^{1}$-transgression The Grothendieck construction is a well-known way to "decategorify" certain types of category. In this construction, one considers the set of isomorphism classes of objects together with a sum operation on it, and formally completes this set to a group. In the case of the representation category of a finite group, this gives rise to the free $\mathbb{Z}$-module generated by the irreducible representations of the group $G$. 
However, given a category, there is another way to go down the categorical ladder and this is to take the "dimension" of the category, which here means the set of natural transformations of the identity functor. Note that this could have extra structure, so when the category is linear, the dimension will be a vector space. In the case of the representation category of a finite group, this dimension is identifiable with the set of class functions on the group: indeed here the Grothendieck group $K(\operatorname{Rep}(G))$ embeds, via the character map, as a full lattice in the dimension, so the dimension is the complexification of the Grothendieck group and the two notions of decategorification coincide.

If $I$ denotes the unit interval, then from the point of view of topological quantum field theory, the $(2-m)$-functor associated to $I \times M$ should be the identity functor on the $(2-m)$-category $V(M)$, so as $S^{1} \times M$ is obtained from $I \times M$ by gluing the ends together, $V\left(S^{1} \times M\right)$ should be in some sense the trace of the identity functor on $V(M)$, ie, it should be in some sense the dimension of the $(2-m)$-category $V(M)$ : the $(1-m)$-category of natural transformations on the identity is a good candidate for this dimension. Now if $\Pi_{1}(M)$ is a finite model for the fundamental groupoid of $M$ then $\overline{\mathbb{Z}} \times \Pi_{1}(M)$ is a finite model for the fundamental groupoid of $S^{1} \times M$. For any finite groupoid $\mathcal{G}$ we can define the loop groupoid (or inertia groupoid) $\Lambda \mathcal{G}$ to be $\mathcal{F}$ un $(\overline{\mathbb{Z}}, \mathcal{G})$. Then

$$
\begin{aligned}
\mathfrak{G}\left(S^{1} \times M\right) & =\mathcal{F} \operatorname{un}\left(\Pi_{1}\left(S^{1} \times M\right), \bar{G}\right)=\mathcal{F} \operatorname{un}\left(\overline{\mathbb{Z}} \times \Pi_{1}(M), \bar{G}\right) \\
& =\mathcal{F} \operatorname{un}\left(\overline{\mathbb{Z}}, \mathcal{F} \operatorname{un}\left(\Pi_{1}(M), \bar{G}\right)\right)=\Lambda \mathfrak{G}(M) .
\end{aligned}
$$

Furthermore as $\tau_{\left(S^{1} \times M\right)} \omega=\tau_{S^{1}} \tau_{M} \omega$, we can write

$$
V\left(S^{1} \times M\right)=\Gamma_{\Lambda \mathfrak{G}(M)}\left(\tau_{S^{1}}\left(\tau_{M} \omega\right)_{\mathbb{C}}\right)
$$

and so we expect this to be the dimension of $V(M)=\Gamma_{\mathfrak{G}(M)}\left(\left(\tau_{M} \omega\right)_{\mathbb{C}}\right)$.

Actually, given what we will see below, we might expect something more general to be the case. Thinking of our model of gerbes to be simplicial cocycles, in Section 1.3.3 we define the general $S^{1}$-transgression map $\tau: Z^{n}(\mathcal{G}, U(1)) \rightarrow Z^{n-1}(\Lambda \mathcal{G}, U(1))$, then we will see in the sections below that for any finite groupoid $\mathcal{G}$ and any one- or two-cocycle $\xi$ on $\mathcal{G}$ we have

$$
\operatorname{dim}\left(\Gamma_{\mathcal{G}}\left(\xi_{\mathbb{C}}\right)\right) \cong \Gamma_{\Lambda \mathcal{G}}\left(\tau(\xi)_{\mathbb{C}}\right)
$$

At this stage this just looks like a not-very meaningful collection of symbols, so it is worth unpacking it a little in the case that $\xi$ is of degree one or two.

When $\xi=\alpha$ is a one-cocycle on $\mathcal{G}$ then $\alpha$ can be interpreted as a $U(1)$-bundle on $\mathcal{G}$, and the space of sections $\Gamma_{\mathcal{G}}(\alpha)_{\mathbb{C}}$ is the space of (flat) sections of the associated 
complex line bundle. The transgression $\tau(\alpha)$ is a locally constant $U(1)$-valued function on the loop groupoid $\Lambda \mathcal{G}$ and the "space of sections" $\Gamma_{\Lambda \mathcal{G}} \tau(\alpha)_{\mathbb{C}}$ is just the integral of $\tau(\alpha)$ over the loop groupoid, and does indeed give the dimension of the vector space $\Gamma_{\mathcal{G}}(\alpha)_{\mathbb{C}}$. In this case we can write $(\star)$ as

$$
\operatorname{dim}\left(\text { sections of the line bundle } \alpha_{\mathbb{C}} \text { over } \mathcal{G}\right)=\int_{\Lambda \mathcal{G}} \tau(\xi),
$$

and this is Theorem 6 .

When $\xi=\theta \in Z^{2}(\mathcal{G}, U(1))$ is a two-cocycle, then the gerbe $\theta$ can be thought of as an central extension of $\mathcal{G}$ by $U(1)$, and the "space of sections" $\Gamma_{\mathcal{G}}(\theta)_{\mathbb{C}}$ is the category of $\theta$-twisted representations of $\mathcal{G}$, whereas $\Gamma_{\Lambda \mathcal{G}}\left(\tau(\theta)_{\mathbb{C}}\right)$ is the vector space of $\theta$-twisted characters, so the assertion comes down to the analogue of the usual statement for finite groups: namely that natural transformations of the identity functor on the category of representations are given precisely by class functions. This analogue is Theorem 16. It is also shown in Theorem 11 that just as in the case of representations of finite groups, the complexified Grothendieck group is isomorphic to the space of twisted characters.

Note that when the groupoid $\mathcal{G}$ is just a group $\bar{G}$ and the two-cocycle $\theta$ is trivial, the above is saying that the characters of $G$ classify the representations and span the class functions, in other words the character map induces an isomorphism

$$
\operatorname{Rep}(G) \otimes \mathbb{C} \cong\{\text { class functions } G \rightarrow \mathbb{C}\} .
$$

Dijkgraaf-Witten theory and the Drinfeld double We can see how the above ideas of transgression and dimension fit into the picture of Dijkgraaf-Witten theory in low dimension. Table 1 gives an indication of what is going on. We assume a fixed finite group $G$ and three-cocycle $\omega \in Z_{\mathrm{gp}}^{3}(G, U(1))$.

By an $\omega$-twisted elliptic character of $G$ I mean a complex-valued function $\chi$, defined on pairs of commuting elements of $G$, which satisfies the following conjugation relation for any $h \in G$ :

$$
\chi\left(h g h^{-1}, h x h^{-1}\right)=\frac{\omega(h, x, g) \omega\left(h g h^{-1}, h, x\right) \omega\left(h x h^{-1}, h g h^{-1}, h\right)}{\omega(h, g, x) \omega\left(h x h^{-1}, h, g\right) \omega\left(h g h^{-1}, h x h^{-1}, h\right)} \chi(g, x) .
$$

Note that in the final column of spaces of sections, by the two instances of the dimension relation $(\star$ ) alluded to above, moving down from the second row to the bottom row, or from the third row to the second, is just the taking the dimension. In the untwisted case, in work with Bruce Bartlett [3] the 2-category of 2-representations of a finite group is defined and it is shown that taking its dimension is just moving down from the top row to the second row. 


\begin{tabular}{|c|c|c|c|}
\hline $\begin{array}{c}\text { manifold } \\
\qquad M\end{array}$ & $\begin{array}{l}\text { groupoid } \\
\mathfrak{G}(M)\end{array}$ & $\begin{array}{l}\text { gerby object } \\
\tau_{M} \omega\end{array}$ & $\begin{array}{c}\text { "space of sections" } \\
\Gamma_{\mathfrak{G}(M)}\left(\left(\tau_{M} \omega\right)_{\mathbb{C}}\right)\end{array}$ \\
\hline$\{*\}$ & $\bar{G}$ & $\begin{array}{c}\omega \\
\text { two-gerbe }\end{array}$ & $\begin{array}{c}i^{2} \text {-category of } \omega \text {-twisted } \\
2 \text {-representations of } \mathrm{G} ?\end{array}$ \\
\hline$S^{1}$ & $\Lambda \bar{G}$ & $\begin{array}{l}\tau(\omega) \\
\text { gerbe }\end{array}$ & $\begin{array}{c}\text { category of representations } \\
\text { of } D^{\omega}(G)\end{array}$ \\
\hline $\mathbb{T}^{2}$ & $\Lambda^{2} \bar{G}$ & $\begin{array}{c}\tau^{2}(\omega) \\
U(1) \text {-bundle }\end{array}$ & $\begin{array}{l}\text { vector space of } \omega \text {-twisted } \\
\text { elliptic characters of } G\end{array}$ \\
\hline $\mathbb{T}^{3}$ & $\Lambda^{3} \bar{G}$ & $\begin{array}{c}\tau^{3}(\omega) \\
U(1) \text {-function }\end{array}$ & $\begin{array}{c}\text { number of } \omega \text {-twisted } \\
\text { elliptic characters of } G\end{array}$ \\
\hline
\end{tabular}

Table 1: Gerbe transgression and Dijkgraaf-Witten theory in low dimensions

\section{The inertia groupoid versus the loop groupoid}

In this paper the terms inertia groupoid and loop groupoid are used interchangeably as we are in the context of finite groupoids. In more general situations this is not the case. A more general class of groupoids is the class of orbifold groupoids which arise, not surprisingly in the study of orbifolds. If $G$ is an orbifold groupoid then, following Haefliger and others, the loop groupoid LG was defined by Lupercio and Uribe [13]. The classifying space of this is homotopy equivalent to the free loop space on the classifying space of the original groupoid: $\mathcal{B L G} \stackrel{\sim}{\longrightarrow} \mathcal{L B G}[17$, Theorem 2.1.1]. The inertia groupoid can be identified as an $S^{1}$-invariant part inside the loop groupoid: $\Lambda \mathrm{G} \simeq(\mathrm{LG})^{S^{1}} \subset \mathrm{LG}$. They also showed, in [15], that the classifying space on the inertia groupoid of $\mathrm{G}$ is homotopy equivalent to a certain ghost loop space on the classifying space of the original groupoid: $\mathcal{B} \Lambda \mathrm{G} \stackrel{\sim}{\longrightarrow} \mathcal{L}_{s} \mathcal{B G}$. Furthermore they gave, in [16], a transgression map in Deligne cohomology; the transgression given in the current paper can be seen as a baby case of that. The interested reader is enthusiastically directed towards the work of Lupercio and Uribe.

\section{What is in this paper}

Section 1 is the geometric part of the paper. It starts at the beginning with preliminaries on classifying spaces of groupoids and cocycles on groupoids. The intention is to make 
this accessible to people who are not familiar with these ideas. Section 1.3 is the heart of the geometric part; in it the loop groupoid of a groupoid is defined, and the Parmesan map, between the classifying space of the loop groupoid and the free loop space on the classifying space of the original groupoid, is constructed. Theorem 2 states that this is a homotopy equivalence. This is then used to give an explicit formula for the loop transgression map on the level of cocycles, $\tau: Z^{n}(\mathcal{G}, U(1)) \rightarrow Z^{n-1}(\Lambda \mathcal{G}, U(1))$, which can be thought of as mapping (flat) $(n-1)$-gerbes on a groupoid to (flat) $(n-2)$-gerbes on its loop groupoid.

Section 2 is the algebraic part of the paper. This is where the theory of twisted representations of groupoids is developed from the point of view of gerbes. First of all there is a quick reminder on the theory of twisted representations of finite groups. Then for a finite groupoid $\mathcal{G}$, considering $n=0,1,2$ in turn, I show how a normalized $n$-cocycle in $Z^{n}(\mathcal{G}, U(1))$ can be thought of as an $(n-1)$-gerbe on $\mathcal{G}$, ie, respectively a $U(1)$-valued function, a principal $U(1)$-bundle and a $U(1)$-gerbe, and how its "complexification" has a "pushforward to a point" which is an $(n-1)$-category, these are respectively the integral over $\mathcal{G}$, the space of sections of the associated complex line bundle, and the category of twisted representations. Furthermore, for $n=1,2, \mathrm{I}$ show that the decategorification of the "space of sections", ie, the dimension or the complexification of the Grothendieck group, is given by the space of sections the transgressed $(n-2)$-gerbe over the loop groupoid. For $n=2$ this generalizes the fact that the space of class functions on a finite group is the complexification of the Grothendieck group of the category of representations. The final subsection shows how the group of twisted representations of a groupoid decomposes into the twisted representation groups of its automorphism groups of its components-this is essentially a version of Dijkgraaf-Pasquier-Roche induction.

Section 3 is where the above work is put to use in the context of the twisted Drinfeld double of a finite group. For $G$ a finite group and $\omega \in H^{3}(G, U(1))$ the "level" or "twisting", it is shown that $D^{\omega}(G)$ is just ${ }^{\tau(\omega)} \mathbb{C} \Lambda \bar{G}$ the twisted groupoid algebra of the loop groupoid on $G$. The representations of this are then the same as the $\tau(\omega)-$ twisted representations of the loop groupoid $\Lambda \bar{G}$ and all of the previous machinery can be applied to see such things as the semisimplicity of $D^{\omega}(G)$, Dijkgraaf-PasquierRoche induction, the fact that its representation group is a twisted equivariant K-group ${ }^{\tau(\omega)} K_{G}\left(G^{\mathrm{c}}\right)$, and the fact that its representations are classified by their characters, the characters being identifiable with "twisted elliptic characters on $G$ ".

Acknowledgements I would like to thank the following people for various enlightening conversations: Mark Brightwell, Jørgen Ellegaard Andersen, Dan Freed, John Greenlees, Kirill Mackenzie, Ieke Moerdijk, Michael Murray, Elmer Rees, Justin 
Roberts, Joost Slingerland, Neil Strickland and Paul Turner. The nomenclature is due to Olivia Rossi-Doria.

\section{Groupoids, classifying spaces and cohomology}

The goal of this section is to give an explicit formula for the loop-transgression map $Z^{*}(\mathcal{G}, U(1)) \rightarrow Z^{*-1}(\Lambda \mathcal{G}, U(1))$. This describes the twisting in the twisted Drinfeld double and also is useful in describing characters of twisted representations of groups.

In Section 1.1 the classifying space of a category is introduced using the geometric bar construction, rather than the usual common barycentric version, as this is more useful in the formulas derived later. In Section 1.2.2 an explicit formula for the well-known fact that a groupoid is equivalent to the union of its automorphism groups is given, as this will be useful. In Section 1.3 the loop groupoid (or inertia groupoid) of a finite groupoid and the Parmesan construction are introduced, the latter being a homotopy equivalence $\mathcal{B} \Lambda \mathcal{G} \rightarrow \mathcal{L B G}$ from the classifying space of the loop groupoid to the free loop space of the classifying space of the original groupoid. From this it is straight forward to write down the transgression map $Z^{n}(\mathcal{G}, U(1)) \rightarrow Z^{n-1}(\Lambda \mathcal{G}, U(1))$. To prove that the Parmesan map is a homotopy equivalence it suffices to prove it for finite groups, this is the content of Section 1.4. The key here is that the loop groupoid $\Lambda \bar{G}$ is also the action groupoid of $G$ on itself by conjugation.

Note that although it was known to the experts that there is a homotopy equivalence $\mathcal{B} \Lambda \mathcal{G} \rightarrow \mathcal{L B G}$, we will need to construct a specific map so that the transgression gives rise exactly to the expression in the product of the twisted Drinfeld double. This, together with the fact that several readers will not be familiar with classifying spaces, is why some detail is gone into here.

\subsection{The classifying space of a finite groupoid}

Recall firstly that a finite groupoid is a category with a finite number of objects and morphisms, in which all of the morphisms are invertible. A good example to bear is mind has one object, and its set of morphisms form a finite group $G$ : this groupoid will be denoted $\bar{G}$.

Given a finite groupoid $\mathcal{G}$, we will associate to it a topological space $\mathcal{B G}$, its classifying space. This is formed by gluing simplices together. (I will be thinking geometrically and blur the distinction between a simplicial complex and its geometric realization.) The $n$-simplices of the classifying space $\mathcal{B G}$ are labelled by the strings of $n$ composable morphisms

$$
x_{n} \stackrel{g_{n}}{\longleftarrow} \ldots \stackrel{g_{2}}{\longleftarrow} x_{1} \stackrel{g_{1}}{\longleftarrow} x_{0} .
$$


(Note that I sometimes write arrows "backward", the reason being that notationally it makes composition behave better.) The corresponding $n$-simplex is thought of as an $n$-simplex in $n$-space with the vertices labelled by $x_{0}, \ldots, x_{n}$, with the directed edge from $x_{i-1}$ to $x_{i}$ of length one, parallel to the $i$-th axis and labelled by $g_{i}$, with the other edges labelled so as to make the two-faces commutative triangles. A three-simplex is pictured in Figure 1. The classifying space $\mathcal{B G}$ is then obtained by gluing common faces together.

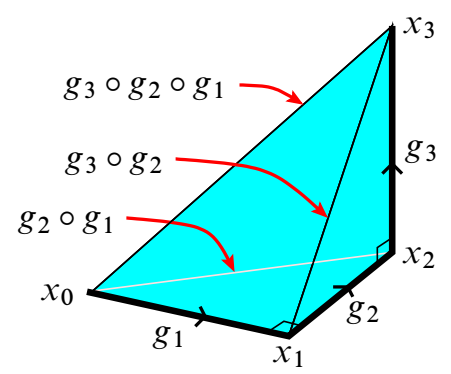

Figure 1: The three-simplex in the classifying space $\mathcal{B G}$ corresponding to $x_{3} \stackrel{g_{3}}{\leftarrow} x_{2} \stackrel{g_{2}}{\longleftarrow} x_{1} \stackrel{g_{1}}{\leftarrow} x_{0}$ This means that a point in the classifying space is given by a string

$$
x_{n} \stackrel{g_{n}}{\longleftarrow} \ldots \stackrel{g_{2}}{\longleftarrow} x_{1} \stackrel{g_{1}}{\longleftarrow} x_{0}
$$

and some coordinates $0 \leq t_{n} \leq \cdots \leq t_{1} \leq 1$ in the simplex; this point will be denoted

$$
\left[g_{n}, t_{n}|\ldots| g_{2}, t_{2} \mid g_{1}, t_{1}\right] \text {. }
$$

The gluing of faces means that there is the following identification of points in $\mathcal{B G}$.

$$
\begin{aligned}
{\left[g_{n}, t_{n}\left|g_{n-1}, t_{n-1}\right| \ldots \mid g_{1}, 1\right] } & =\left[g_{n}, t_{n}|\ldots| g_{2}, t_{2}\right] ; \\
{\left[g_{n}, t_{n}|\ldots| g_{i+1}, t\left|g_{i}, t\right| \ldots \mid g_{1}, t_{1}\right] } & =\left[g_{n}, t_{n}|\ldots| g_{i+1} \circ g_{i}, t|\ldots| g_{1}, t_{1}\right] ; \\
{\left[g_{n}, 0\left|g_{n-1}, t_{n-1}\right| \ldots \mid g_{1}, t_{1}\right] } & =\left[g_{n-1}, t_{n-1}|\ldots| g_{1}, t_{1}\right] .
\end{aligned}
$$

This is just saying that a point is in the face of a simplex when two adjacent coordinates are equal, when the first coordinate is 1 or when the final coordinate is 0 .

I think it is more common to see barycentric coordinates used for simplices, but the coordinate system given here will have advantages when prisms are decomposed below. Another common variation is the imposition of degeneracy conditions, so that an $n$-simplex in which one of the morphisms is an identity morphism, is identified with the $(n-1)$-simplex obtained by removing that morphism. I do not impose these conditions as it is not necessary. However, when considering cocycles it will be convenient to consider normalized cocycles, these are the ones which vanish on 
simplices containing an identity morphism (see below). The normalized and nonnormalized cochain complexes are homotopic and so have the same cohomology groups, thus it is primarily a matter of convenience as to which is used.

Any functor $F: \mathcal{G} \rightarrow \mathcal{H}$ between finite groupoids determines a map $\mathcal{B} F: \mathcal{B G} \rightarrow \mathcal{B H}$ between classifying spaces given by

$$
\mathcal{B F}:\left[g_{n}, t_{n}|\ldots| g_{2}, t_{2} \mid g_{1}, t_{1}\right] \mapsto\left[F\left(g_{n}\right), t_{n}|\ldots| F\left(g_{2}\right), t_{2} \mid F\left(g_{1}\right), t_{1}\right] .
$$

By definition, the cohomology of $\mathcal{G}$ is taken to be the simplicial cohomology of $\mathcal{B G}$ (thought of as a simplicial complex), so an $n$-cochain on $\mathcal{G}$ means a function from the set of $n$-simplices of $\mathcal{B G}$, which will be normalized so that $\left[g_{n}|\ldots| \operatorname{Id}_{x_{i}}|\ldots| g_{1}\right]$ maps to the unit. Furthermore $\omega$ is an $n$-cocycle if it is an $n$-cochain which vanishes on boundaries, so that

$$
\begin{aligned}
& \omega\left(\left[g_{n-1}|\ldots| g_{1}\right]\right) \omega\left(\left[g_{n} g_{n-1}|\ldots| g_{1}\right]\right)^{-1} \\
& \cdots \omega\left(\left[g_{n}|\ldots| g_{2} g_{1}\right]\right)^{ \pm 1} \omega\left(\left[g_{n}|\ldots| g_{2}\right]\right)^{\mp 1}=1 .
\end{aligned}
$$

If $G$ is a finite group then $\bar{G}$ was defined to be the category with one object, with the morphism set being $G$ and with composition being multiplication in $G$. Then $\mathcal{B} \bar{G}$ is a model for the classifying space for $G$ so that $H^{*}(\bar{G})$ is the usual group cohomology of $G$. This particular model is known as the geometric bar construction (see Gajer [10]) and it is originally due to Milgram [18]. One of the advantages of this over the usual Milnor construction is that if $G$ is an abelian group then so is the geometric bar construction, so the process can be iterated.

\subsection{Equivalence of groupoids}

Many of the groupoids under consideration, such as $\mathfrak{G}(M)$ in the introduction, depend on certain choices and are really only defined up to equivalence, and in fact should really be thought of as representing a stack. Equivalence of groupoids translates to homotopy equivalence of the corresponding classifying spaces. This means that the constructions here ought to be natural with respect to such equivalences. This does have the advantage that certain properties can just be proved for groups as it is shown below that groupoids are equivalent to the disjoint union of groups.

1.2.1 Natural transformations and homotopies If $F$ and $F^{\prime}$ are both functors from $\mathcal{G}$ to $\mathcal{H}$, then a natural transformation, $T$, from $F$ to $F^{\prime}$, written $T: F \Rightarrow F^{\prime}$ consists of a morphism $T(x): F(x) \rightarrow F^{\prime}(x)$ for every object $x$ such that for every morphism $g: x_{1} \rightarrow x_{2}$ in $\mathcal{G}$ we have $F^{\prime}(g) \circ T\left(x_{1}\right)=T\left(x_{2}\right) \circ F(g)$, so $T$ intertwines 
the two functors. Two functors between groupoids are said to be isomorphic if there is a natural transformation between them.

Given such a natural transformation $T: F \Rightarrow F^{\prime}$ we get a homotopy $H_{T}$ between the classifying maps $\mathcal{B F}$ and $\mathcal{B} F^{\prime}$, in other words we get a map $H_{T}: \mathcal{B G} \times[0,1] \rightarrow \mathcal{B H}$ such that $H_{T}(-, 0)=F(-)$ and $H_{T}(-, 1)=F^{\prime}(-)$. Explicitly it can be written as

$$
\begin{aligned}
& H_{T}\left(\left[g_{n}, t_{n}|\ldots| g_{1}, t_{1}\right], t\right) \\
& \quad:=\left[F^{\prime}\left(g_{n}\right), t_{n}|\ldots| F^{\prime}\left(g_{i+1}\right), t_{i+1}\left|T\left(x_{i}\right), t\right| F\left(g_{i}\right), t_{i}|\ldots| F\left(g_{1}\right), t_{1}\right] \\
& \quad \text { for } 0 \leq t_{n} \leq \ldots \leq t_{i+1} \leq t \leq t_{i} \leq \ldots t_{1} \leq 1 .
\end{aligned}
$$

This formula should be made clearer by looking at Figure 2, which is where the formula comes from.
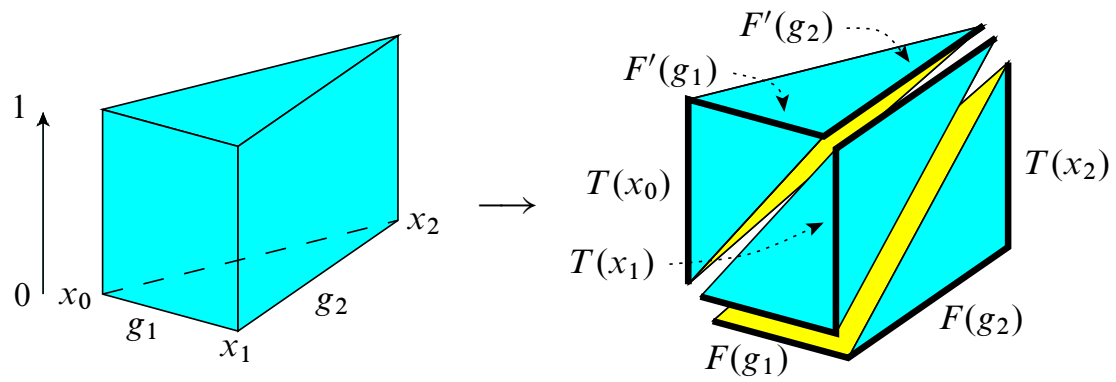

Figure 2: The homotopy construction

1.2.2 Equivalence of groupoids to unions of groups Two groupoids $\mathcal{G}$ and $\mathcal{H}$ are said to be equivalent if there are functors $F: \mathcal{G} \rightarrow \mathcal{H}$ and $\bar{F}: \mathcal{H} \rightarrow \mathcal{G}$ such that $F \circ \bar{F}$ is isomorphic to the identity functor on $\mathcal{H}$ and $\bar{F} \circ F$ is isomorphic to the identity functor on $\mathcal{G}$. Equivalent groupoids share many features, such as having the same representation theory, having homotopic classifying spaces $(\mathcal{B} F$ and $\mathcal{B} \bar{F}$ supplying the homotopy equivalence), and having isomorphic homology and cohomology groups.

A fundamental feature of groupoids is that every groupoid is equivalent to a disjoint union of groups, thus as we will see, many problems about groupoids reduce to problems about groups. As this will be so useful, I will give the details here.

To see this equivalence, it suffices to consider connected groupoids and to show that every connected groupoid is equivalent to a groupoid with one object, ie, a group. So given a connected groupoid $\mathcal{G}$ fix an object $x$, and define the automorphism group $\operatorname{Aut}(x)$ of $x$ to be the group of morphisms $\operatorname{Mor}_{\mathcal{G}}(x, x)$. Let $\overline{\operatorname{Aut}(x)}$ be the groupoid with the single object, $x$, and the morphism group $\operatorname{Aut}(x)$. Define $i: \overline{\operatorname{Aut}(x)} \rightarrow \mathcal{G}$ 
to be the inclusion. To construct an inverse equivalence to $i$ we need to make some choices: for each object $y$ in $\mathcal{G}$ pick a morphism $f_{y}: y \rightarrow x$, taking $f_{x}: x \rightarrow x$ to be the identity morphism. Now define the "retract" $r: \mathcal{G} \rightarrow \overline{\operatorname{Aut}(x)}$ by

$$
\begin{array}{ll}
r(y):=x & \text { for every object } y \\
r(g):=f_{z} \circ g \circ f_{y}^{-1} & \text { for every morphism } g: y \rightarrow z .
\end{array}
$$

To see that this gives an equivalence of groupoids, we just note that $r \circ i=\operatorname{Id}$ and that a natural isomorphism $T: \operatorname{Id}_{\mathcal{G}} \Rightarrow i \circ r$ is given by defining $T(y):=f_{y}$ for every object $y$.

From this the disconnected case easily follows:

Theorem 1 If $\mathcal{G}$ is a groupoid then $\mathcal{G}$ is equivalent to the groupoid $\bigsqcup_{x} \overline{\operatorname{Aut}(x)}$ where $x$ runs over one object from each connected component of $\mathcal{G}$.

\subsection{Loop groupoids, the Parmesan Theorem and transgression}

This is the heart of this section on classifying spaces. Firstly in Section 1.3.1 given a finite groupoid $\mathcal{G}$ the key notion of its loop groupoid $\Lambda \mathcal{G}$ is introduced. Then in Section 1.3.2 the Parmesan construction is described, this is a homotopy equivalence $\mathcal{B} \Lambda \mathcal{G} \rightarrow \mathcal{L B G}$ where $\mathcal{L B G}$ is the free loop space on the classifying space $\mathcal{B G}$. In Section 1.3.3 this leads to the $S^{1}$-transgression map on the level of cocycles $\tau: Z^{*}(\mathcal{G}, U(1)) \rightarrow$ $Z^{*-1}(\Lambda \mathcal{G}, U(1))$, which will play a leading role in Section 2. In Section 1.3.4 another homotopy equivalence $\mathcal{B} \Lambda \mathcal{G} \rightarrow \mathcal{L B G}$, essentially due to Burghelea, is mentioned.

1.3.1 Loop groupoids For a finite groupoid $\mathcal{G}$ define the loop groupoid (also known as the inertia groupoid) $\Lambda \mathcal{G}$ as follows. The objects of $\Lambda \mathcal{G}$ are the self morphisms of $\mathcal{G}$, ie, $\amalg_{x \in O b \mathcal{G}} \operatorname{Mor}_{\mathcal{G}}(x, x)$, and there is a morphism $g_{\gamma}: \gamma \rightarrow g \gamma g^{-1}$ in $\Lambda \mathcal{G}$ for each $\gamma \in \operatorname{Mor}_{\mathcal{G}}(x, x)$ and $g \in \operatorname{Mor}_{\mathcal{G}}(x, y)$-by abuse of notation I will usually denote $g_{\gamma}$ it by $g$ when the source is clear. Composition is induced from that in $\mathcal{G}$. An example is pictured in Figure 3.

An equivalent way to define $\Lambda \mathcal{G}$, and the point of view taken in the introduction, is as $\mathcal{F}$ un $(\overline{\mathbb{Z}}, \mathcal{G})$, the functor category to $\mathcal{G}$ from the one object category with morphism group $\mathbb{Z}$, with the morphisms in the functor category being given by natural transformations.

As the name suggests this is supposed to be a model for "loops on $\mathcal{G}$ ", actually it is a model for loops on $\mathcal{B G}$, as shown below. This should appear reasonable from the second description in terms of the functor category as it is asserting that there is a 


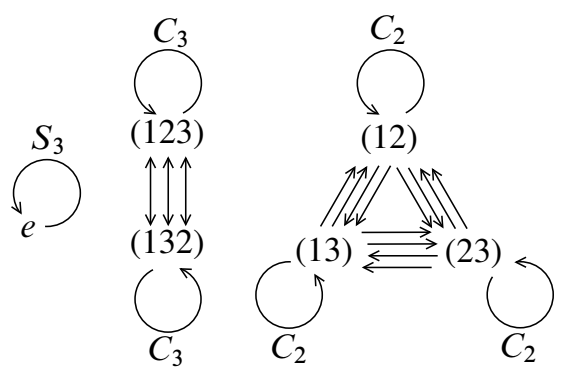

Figure 3: The loop groupoid $\Lambda \overline{S_{3}}$ or equivalently the action groupoid $\mathcal{G}_{S_{3}}\left(S_{3}^{c}\right)$ for $S_{3}$ with the conjugation action (where $S_{3}$ denotes the symmetric group on three letters). The automorphism groups have been marked on.

homotopy equivalence $\mathcal{B} \mathcal{F}$ un $(\overline{\mathbb{Z}}, \mathcal{G}) \simeq \operatorname{Maps}\left(S^{1}, \mathcal{B G}\right)$ and it is a standard fact that $\mathcal{B} \overline{\mathbb{Z}}$ is homotopy equivalent to a circle. This homotopy equivalence is reasonably well known — see Lupercio and Uribe [15] and references therein, also Strickland [19]—but we will need a certain explicit form of the map here.

1.3.2 The Parmesan Theorem For simplicity of notation I will denote the $n$-simplex in $\Lambda \mathcal{G}$

$$
g_{n} \ldots g_{1} \gamma g_{1}^{-1} \ldots g_{n}^{-1} \stackrel{g_{n}}{\leftarrow} \ldots \stackrel{g_{1}}{\leftarrow} \gamma \quad \text { by } \quad\left[g_{n}|\ldots| g_{1}\right] \gamma .
$$

We now get to the key construction. Denote by $\mathcal{L B G}$ the free loop space on $\mathcal{B G}$, in other words $\mathcal{L B G}:=\operatorname{Maps}\left(S^{1}, \mathcal{B G}\right)$, and define the Parmesan map Par: $\mathcal{B} \Lambda \mathcal{G} \rightarrow \mathcal{L B G}$ by

$$
\begin{aligned}
& \operatorname{Par}\left(\left[g_{n}, t_{n}|\ldots| g_{1}, t_{1}\right] \gamma\right)(t) \\
& \quad=\left[g_{n}, t_{n}|\ldots| g_{i+1}, t_{i+1}\left|\left(g_{i} \ldots g_{1}\right) \gamma\left(g_{i} \ldots g_{1}\right)^{-1}, t\right| g_{i}, t_{i}|\ldots| g_{1}, t_{1}\right] \\
& \quad \text { for } t_{i} \geq t \geq t_{i+1} .
\end{aligned}
$$

This is illustrated graphically in Figure 4, which is where it gets its name from.

Note that it was precisely to get this simple formula that I used these coordinates on the simplices. The key result about this map is the following.

Theorem 2 If $\mathcal{G}$ is a finite groupoid and $\Lambda \mathcal{G}$ is its loop groupoid then the Parmesan map Par: $\mathcal{B} \Lambda \mathcal{G} \rightarrow \mathcal{L B G}$ is a homotopy equivalence.

Proof The main point in the proof is that we first prove it for finite groups and then we prove it for general finite groupoids. The statement of the theorem for finite groups is Theorem 4, which is proved below. So we can assume it is true for a finite group. Now assume that $\mathcal{G}$ is a connected, finite groupoid-the result in the nonconnected case 

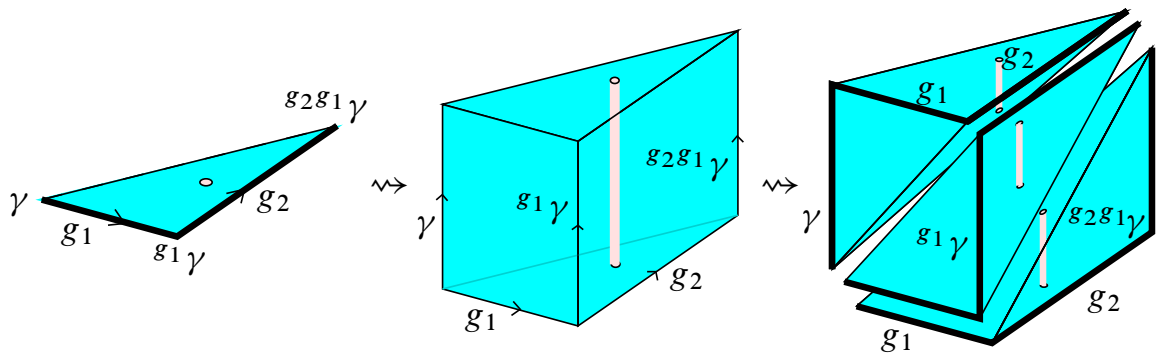

Figure 4: The parmesan construction mapping a point in a two-simplex of $\mathcal{B} \Lambda \mathcal{G}$ to a loop in $\mathcal{B G}$. For reasons of space, here ${ }^{g} \gamma$ denotes $g \gamma g^{-1}$.

follows from this. Pick any object $x$ in $\mathcal{G}$ and let $G$ be the automorphism group of $x$. By Section 1.2.2, the inclusion $\bar{G} \rightarrow \mathcal{G}$ gives equivalence of groupoids, so the induced inclusion $\mathcal{B} \bar{G} \rightarrow \mathcal{B G}$ is a homotopy equivalence, and as the loop space functor is a homotopy functor, the inclusion $\mathcal{L B} \bar{G} \rightarrow \mathcal{L B \mathcal { G }}$ is a homotopy equivalence. Similarly the inclusion $\Lambda \bar{G} \rightarrow \Lambda \mathcal{G}$ is an equivalence, implying that the inclusion $\mathcal{B} \Lambda \bar{G} \rightarrow \mathcal{B} \Lambda \mathcal{G}$ is a homotopy equivalence.

It is immediate that the following diagram commutes

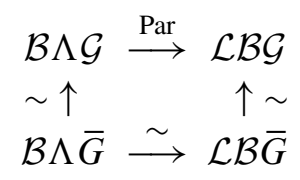

and as the three marked maps are homotopy equivalences, it follows that the Parmesan map must be as well.

1.3.3 The transgression map As we have the map $\mathcal{B} \Lambda \mathcal{G} \rightarrow \mathcal{L B \mathcal { G }}$ we also have the adjoint map (which can be thought of as the evaluation map) $\mathcal{B} \Lambda \mathcal{G} \times S^{1} \rightarrow \mathcal{B G}$ and thus the map on the level of chains $C_{n}(\Lambda \mathcal{G}, \mathbb{Z}) \rightarrow C_{n+1}(\mathcal{G}, \mathbb{Z})$ which is given by

$\left[g_{n}|\ldots| g_{1}\right] \gamma \mapsto \sum_{i=0}^{n}(-1)^{n-i-1}\left[g_{n}|\ldots| g_{i+1}\left|\left(g_{i} \ldots g_{1}\right) \gamma\left(g_{i} \ldots g_{1}\right)^{-1}\right| g_{i}|\ldots| g_{1}\right]$.

Apart from the signs, this is seen for the case $n=2$ in Figure 4 .

Dualizing this and taking $U(1)$-coefficients, we get the transgression map on the level of cocycles: 
Theorem 3 For a finite groupoid $\mathcal{G}$ the $S^{1}$-transgression map on the cocycle level, $\tau: Z^{*}(\mathcal{G}, U(1)) \rightarrow Z^{*-1}(\Lambda \mathcal{G}, U(1))$, is given on a cocycle $\alpha \in Z^{n}(\mathcal{G}, U(1))$ by

$$
\begin{aligned}
& \tau(\alpha)\left(\left[g_{n-1}|\ldots| g_{1}\right] \gamma\right):= \\
& \quad \prod_{i=0}^{n-1} \alpha\left(\left[g_{n-1}|\ldots| g_{i+1}\left|\left(g_{i} \ldots g_{1}\right) \gamma\left(g_{i} \ldots g_{1}\right)^{-1}\right| g_{i}|\ldots| g_{1}\right]\right)^{(-1)^{n-1-i}}
\end{aligned}
$$

We will use this for one-cocycles, two-cocycles and three-cocycles, so it is worth explicitly recording them here. So supposing that $\omega$ is a three-cocycle, $\theta$ is a twococycle and $\alpha$ is a one-cocycle, then

$$
\begin{gathered}
\tau(\alpha)([] \gamma):=\alpha([\gamma]) ; \quad \tau(\theta)([g] \gamma):=\frac{\theta\left(\left[g \gamma g^{-1} \mid g\right]\right)}{\theta([g \mid \gamma])} ; \\
\tau(\omega)([h \mid g] \gamma):=\frac{\omega([h|g| \gamma]) \omega\left(\left[h g \gamma g^{-1} h^{-1}|h| g\right]\right)}{\omega\left(\left[h\left|g \gamma g^{-1}\right| g\right]\right)} .
\end{gathered}
$$

The latter formula should look familiar to the reader au fait with the twisted Drinfeld double of a finite group.

1.3.4 Another map to the loop space It was pointed out by an anonymous referee that another homotopy equivalence from the classifying space of the inertia groupoid to the loop space on the classifying space of the original groupoid can be obtained via the work of Lupercio and Uribe [15] which follows on from ideas of Burghelea, Segal and McCord. The idea is that the classifying space of the inertia groupoid is homeomorphic to the cyclic nerve of the groupoid which is homeomorphic to a certain configuration space of points on the circle which maps to the free loop space of a certain configuration space of points on the unit interval which is homeomorphic to the free loop space on the classifying space of the original groupoid. It appears that this alternative map Alt: $\mathcal{B} \Lambda \mathcal{G} \rightarrow \mathcal{L B G}$ is given by

$$
\begin{aligned}
& \operatorname{Alt}\left(\left[g_{n}, t_{n}|\ldots| g_{1}, t_{1}\right] \gamma\right)(t)= \\
& {\left[g_{i}, t_{i}-t|\ldots| g_{1}, t_{1}-t\left|\gamma\left(g_{n} \ldots g_{1}\right)^{-1}, 1-t\right| g_{n}, 1+t_{n}-t|\ldots| g_{i+1}, 1+t_{i+1}-t\right]} \\
& \quad \text { for } t_{i} \geq t \geq t_{i+1} .
\end{aligned}
$$

There is a good pictorial interpretation of this and details of it should appear elsewhere.

\subsection{Action groupoids and the Parmesan Theorem for finite groups}

1.4.1 The definition of action groupoids Suppose that $G$ is a finite group and $X$ is a finite $G$-set, so that there is an action $G \times X \rightarrow X,(g, x) \mapsto g \cdot x$. The action 
groupoid $\mathcal{G}_{G}(X)$, which is sometimes denoted $G \times X \rightrightarrows X$, is the groupoid whose objects are elements of $X$, whose morphisms are of the form $g \cdot x \stackrel{g}{\leftarrow} x$, with $x \in X$ and $g \in G$, and where composition is given by the obvious

$$
(h g \cdot x \stackrel{h}{\leftarrow} g \cdot x) \circ(g \cdot x \stackrel{g}{\longleftarrow} x):=(h g \cdot x \stackrel{h g}{\longleftarrow} x) .
$$

We will see below that the invariants of this groupoid are well known things: its classifying space is the Borel construction on $X$, its homology is the equivariant homology of $X$ and its representation group is the equivariant $\mathrm{K}$-theory of $X$. Of course as $X$ is a finite set, these things are not as exciting as they might be, but we do get interesting information as we will discover.

1.4.2 Examples of action groupoids For any finite group $G$ there are three finite $G$-sets that come to mind.

Firstly there is the trivial, one-element $G$-set $\{*\}$. In this case the action groupoid $\mathcal{G}_{G}(\{*\})$ is just $\bar{G}$. Its classifying space is $\mathcal{B} G$, the classifying space of the group.

Secondly there is the $G$-set $G^{L}$, this is $G$ with the left regular action on itself. In this case $\mathcal{G}_{G}\left(G^{L}\right)$ has $G$ as its set of objects and from $g$ to $h$ there is a unique morphism

$$
g \stackrel{h g^{-1}}{\longleftarrow} h .
$$

In particular this means that $\mathcal{G}_{G}\left(G^{L}\right)$ is connected and has trivial automorphism groups, so is equivalent to the trivial groupoid with one object, thus the classifying space is contractible. Furthermore $G$ acts freely on the right on the groupoid $\mathcal{G}_{G}\left(G^{L}\right)$ via

$$
\left(g \stackrel{h g^{-1}}{\longrightarrow} h\right) \cdot k:=g k \stackrel{h g^{-1}}{\longrightarrow} h k .
$$

From this we get a free action of $G$ on the classifying space. We will denote the classifying space $\mathcal{B G}_{G}\left(G^{L}\right)$ by $\mathcal{E} G$. The quotient map by the $G$-action on this classifying space gives a $G$-bundle $\mathcal{E} G \rightarrow \mathcal{B} G$ known as the universal $G$-bundle, it is the classifying map of groupoids coming from the $G$-set map $G^{L} \rightarrow\{*\}$.

Thirdly there is the $G$-set $G^{\mathrm{c}}$, this is $G$ with the conjugation action on itself, ie, $G \times G^{\mathrm{c}} \rightarrow G^{\mathrm{c}}$ is $(g, h) \rightarrow g h g^{-1}$. Unpacking the definition of the action groupoid $\mathcal{G}_{G}\left(G^{\mathrm{c}}\right)$ reveals that it is precisely $\Lambda \bar{G}$, the loop groupoid on the one object category $\bar{G}$. The main result of this section is to show that the classifying space of this is, via the Parmesan map, homotopy equivalent to the loop space on the classifying space $\mathcal{B} G$.

1.4.3 Classifying spaces and the transgression map For a general $G$-set $X$ the classifying space of the action groupoid $\mathcal{G}_{G}(X)$ can be identified with $\mathcal{E} G \times_{G} X$, 
the Borel construction on $X$, as follows. Firstly, to simplify notation, I will denote a general $n$-simplex of $\mathcal{G}_{G}(X)$ of the form

$$
g_{n} \ldots g_{1} \cdot x \stackrel{g_{n}}{\longleftarrow} \ldots \stackrel{g_{2}}{=} g_{1} \cdot x \stackrel{g_{1}}{\longleftarrow} x \quad \text { by } \quad\left[g_{n}|\ldots| g_{1}\right] x .
$$

Recalling that the $\mathcal{E} G$ was defined above to be the classifying space $\mathcal{B G}_{G}\left(G^{L}\right)$, we can define a map

$$
m: \mathcal{E} G \times_{G} X \rightarrow \mathcal{B G}_{G}(X) ; \quad\left(\left[g_{n}|\ldots| g_{1}\right] g, x\right) \mapsto\left[g_{n}|\ldots| g_{1}\right](g \cdot x) .
$$

This is clearly a homeomorphism as an inverse is given by

$$
\left[g_{n}|\ldots| g_{1}\right] x \mapsto\left(\left[g_{n}|\ldots| g_{1}\right] e, x\right) .
$$

where $e$ is the identity in $G$. Thus the classifying space on the action groupoid of $X$ is the Borel construction on $X$.

Two facts worth mentioning here are: firstly, that the equivariant cohomology of $X$ is defined to be the cohomology of the Borel construction, this means that there is an isomorphism $H_{G}^{*}(X) \cong H^{*}\left(\mathcal{G}_{G}(X)\right)$; and secondly, that the equivariant K-theory of $X$ is by definition the Grothendieck group of equivariant bundles on $X$, but an equivariant bundle on $X$ translates into a representation of the action groupoid of $X$, thus $K_{G}(X) \cong \operatorname{Rep}\left(\mathcal{G}_{G}(X)\right)$, and this is not, in general the same thing as the K-theory of the Borel construction.

We are now ready to prove the Parmesan Theorem for a finite group. (Recall that $\mathcal{L B} G$ denotes the free loop space on the classifying space of $G$.)

Theorem 4 If $G$ is a finite group then the Parmesan map Par: $\mathcal{B} \Lambda \bar{G} \rightarrow \mathcal{L B} G$ (constructed in Section 1.3.2) is a homotopy equivalence.

Proof We will show that the Parmesan map is a homotopy equivalence by giving a homotopy inverse

$$
\mathcal{L} \mathcal{B} G \stackrel{\varphi}{\rightarrow} \mathcal{E} G \times{ }_{G} G^{\mathrm{c}} \stackrel{m}{\rightarrow} \mathcal{B} \Lambda \bar{G} .
$$

We have already observed that the loop groupoid $\Lambda \bar{G}$ for a finite group is the same as the action groupoid $\mathcal{G}_{G}\left(G^{\mathrm{c}}\right)$ for $G$ with the conjugation action, so we can take the map $m: \mathcal{E} G \times_{G} G^{\mathrm{c}} \rightarrow \mathcal{B G}_{G}\left(G^{\mathrm{c}}\right)$ to be the homeomorphism defined a couple of paragraphs above.

Define $\varphi: \mathcal{L} \mathcal{B} \bar{G} \rightarrow \mathcal{E} G \times{ }_{G} G^{\mathrm{c}}$ as follows. If $\lambda$ is a loop in $\mathcal{L B} \bar{G}$, thought of as a map $[0,1] \rightarrow \mathcal{B} \bar{G}$ with the endpoints mapping to the same point, then pick a lift of $\lambda(0)$ to the covering space $\mathcal{E} G$. This leads to a lift $\tilde{\lambda}:[0,1] \rightarrow \mathcal{E} G$ of $\lambda$, and as $\tilde{\lambda}(0)$ and $\tilde{\lambda}(1)$ lie in the same fibre, they differ by an element $g_{\tilde{\lambda}} \in G$, so $\left(\tilde{\lambda}(0), g_{\tilde{\lambda}}\right)$ gives an element 
in $\mathcal{E} G \times G$, but this element depended on the lift of $\lambda(0)$, however this ambiguity is precisely absorbed by considering this as an element $\varphi(\lambda)$ of $\mathcal{E} G \times{ }_{G} G^{\mathrm{c}}$. A proof that this map is a homotopy equivalence is given by Benson [4, Lemma 2.12.1].

To show that $m \circ \varphi$ is a homotopy inverse for Par, as $m \circ \varphi$ is a homotopy equivalence, it suffices to show that $m \circ \varphi \circ \operatorname{Par}=\mathrm{Id}$. To do this we calculate $\varphi \circ$ Par. Suppose that $p$ is a point in the classifying space $\mathcal{B} \Lambda \bar{G}$, we can write $p=\left[g_{n}, t_{n}|\ldots| g_{1}, t_{1}\right] \gamma$ for $g_{1}, \ldots, g_{n}, \gamma \in G$. Now $\operatorname{Par}(p)$ is the loop in $\mathcal{B} G$ pictured in Figure 4 with the vertices all implicitly labelled by $*$. We can lift this to a path $\widetilde{\operatorname{Par}(p)}$ in $\mathcal{E} G$ by picking an element $a \in G$ with which to label the bottom left vertex, this induces a labelling on all of the other vertices. For example the vertex above is labelled $\gamma a$ and the one to the right is labelled $g_{1} a$, as $G$ is acting by left translation ( $\mathcal{E} G$ was defined to be $\left.\mathcal{B} \mathcal{G}_{G}\left(G^{L}\right)\right)$. With this choice of lift we see that

$$
\begin{aligned}
& \widetilde{\operatorname{Par}(p)}(0)=\left[g_{n}, t_{n}|\ldots| g_{1}, t_{1}\right] a \\
& \widetilde{\operatorname{Par}(p)}(1)=\left[g_{n}, t_{n}|\ldots| g_{1}, t_{1}\right] \gamma a .
\end{aligned}
$$

Thus

$$
\varphi \circ \operatorname{Par}(p)=\left(\left[g_{n}, t_{n}|\ldots| g_{1}, t_{1}\right] a, a^{-1} \gamma a\right) \in \mathcal{E} G \times_{G} G^{\mathrm{c}} .
$$

Hence, by the definition of $m$, we get $m \circ \varphi \circ \operatorname{Par}(p)=p$ and so $m \circ \varphi$ is a homotopy inverse to Par as required.

Thus in the case of finite group $G$ we get an explicit map $\mathcal{B G}\left(G^{\mathrm{c}}\right) \rightarrow \mathcal{L B} G$. We know that a groupoid is equivalent to the disjoint union of its automorphism groups and in this case the automorphism group of $x \in G^{\mathrm{c}}$ is just its centralizer $C_{x}$ in $G$, that is, the set of elements in $G$ which commute with $x$. Thus we get the standard result that $H^{*}(\mathcal{L B} G, U(1)) \cong \bigoplus H_{\mathrm{gp}}^{*}\left(C_{x}, U(1)\right)$ where $x$ runs over one representative for each conjugacy class. Combining this with the transgression map $H_{\mathrm{gp}}^{*}(G, U(1)) \rightarrow$ $H^{*-1}(\mathcal{L B} G, U(1))$ gives maps $\tau_{x}: H_{\mathrm{gp}}^{*}(G, U(1)) \rightarrow H_{\mathrm{gp}}^{*-1}\left(C_{x}, U(1)\right)$ for any element $x \in G$. As these maps crop up in a few places such as discrete torsion, it is worth writing them down here on the level of cocycles in small degree. If $\theta \in Z_{\mathrm{gp}}^{2}(G, U(1))$ is a group two-cocycle on $G, \omega \in Z_{\mathrm{gp}}^{3}(G, U(1))$ is a three-cocycle, $x \in G$ and $h, g \in C_{x}$ then

$$
\tau_{x} \theta(g):=\frac{\theta(g, x)}{\theta(x, g)} ; \quad \tau_{x} \omega(h, g):=\frac{\omega(h, g, x) \omega(x, h, g)}{\omega(h, x, g)} .
$$

\section{Twisted representations and cohomology}

This section is the algebraic heart of the paper in which the theory of twisted representations of finite groupoids is developed. The philosophy behind it is very much that of 
$n$-gerbes. What this means is that starting with $n=0$ and working up to $n=2$ we see how an $n$-cocycle leads to an $(n-1)$-category which is to be interpreted as the integral or space of sections or pushforward to a point of the 'complexified gadget' associated to the $(n-1)$-gerbe. Furthermore the things at level $(n-1)$ are classified in an appropriate sense by the transgressed $(n-2)$ cocycle over the loop groupoid. All the constructions are natural with respect to equivalences of groupoids.

First here is a quick reminder on the theory of twisted representations of finite groups-a reasonable reference is Karpilovsky's book [12].

\section{Reminder on twisted representations of finite groups}

Suppose that $G$ is a finite group and $\theta \in Z^{2}(G, U(1))$ is a normalized two-cocycle, so it is a $U(1)$-valued function on $G \times G$ satisfying:

$$
\begin{aligned}
\theta(h, g) \theta(k h, g)^{-1} \theta(k, h g) \theta(k, h)^{-1} & =1 & & \text { for all } k, h, g \in G ; \\
\theta(e, g)=\theta(g, e) & =1 & & \text { for all } g \in G .
\end{aligned}
$$

A $\theta$-twisted representation of $G$ (also known as a projective representation with twisting-cocycle $\theta$ ) is defined to be a function $\rho: G \rightarrow \operatorname{End}(V)$ for some vector space $V$ such that

$$
\begin{aligned}
\rho(h) \rho(g) & =\theta(h, g) \rho(h g) ; \\
\rho(e) & =1 .
\end{aligned}
$$

There are a couple of other ways to think of a twisted representation. One way is via the central extension $U(1) \rightarrow{ }^{\theta} G \rightarrow G$, where the underlying set of ${ }^{\theta} G$ is taken as $U(1) \times G$ and the product is taken to be $(z, h)(w, g):=(\theta(h, g) z w, h g)$; a $\theta$-twisted representation of $G$ can then be thought of as a representation of ${ }^{\theta} G$ in which the central $U(1)$ acts in its natural way. Another way to think about a $\theta$-twisted representation is as a representation of the twisted group algebra ${ }^{\theta} \mathbb{C} G$, which is the algebra with basis elements $\{\langle g\rangle\}_{g \in G}$ and with multiplication given by $\langle h\rangle\langle g\rangle:=\theta(h, g)\langle h g\rangle$. To reconcile these two other points of view, note that ${ }^{\theta_{\mathbb{C}}} \cong \mathbb{C} \otimes_{U(1)}{ }^{\theta} G$.

One of the simplest nontrivial examples is the following. Consider the four-group $\mathbb{Z}_{2} \times$ $\mathbb{Z}_{2}=\left\langle a, b \mid a^{2}=b^{2}=(a b)^{2}=1\right\rangle$. Define the two-cocycle $\theta_{V} \in Z^{2}\left(\mathbb{Z}_{2} \times \mathbb{Z}_{2}, U(1)\right)$ by

$$
\theta_{V}\left(a^{u_{a}} b^{u_{b}}, a^{v_{a}} b^{v_{b}}\right)=(-1)^{u_{a} \cdot v_{b}} .
$$

Then the map ${ }^{\theta_{V}} \mathbb{C}\left(\mathbb{Z}_{2} \times \mathbb{Z}_{2}\right) \rightarrow M_{2}(\mathbb{C})$ given by

$$
e \mapsto\left(\begin{array}{ll}
1 & 0 \\
0 & 1
\end{array}\right), \quad a \mapsto\left(\begin{array}{cc}
1 & 0 \\
0 & -1
\end{array}\right), \quad b \mapsto\left(\begin{array}{cc}
0 & i \\
-i & 0
\end{array}\right), \quad a b \mapsto\left(\begin{array}{cc}
0 & -i \\
-i & 0
\end{array}\right),
$$


is an isomorphism.

It is well-known that ordinary representations of finite groups are classified up to equivalence by their character, this is the function on $G$, invariant under conjugation, given on an element by taking the trace of the element in the corresponding representation. Trying the exact same trick with twisted representations does not work in general as taking the trace does not lead to a function on $G$ invariant under conjugation, as the following lemma demonstrates.

Lemma 5 For $\rho$ a $\theta$-twisted representation of the finite group $G$, with $g, h \in G$, the following conjugation relation holds:

$$
\operatorname{Tr}_{\rho}\left(h g h^{-1}\right)=\frac{\theta\left(h g h^{-1}, h\right)}{\theta(h, g)} \operatorname{Tr}_{\rho}(g) .
$$

Proof Firstly using the basic property of $\rho$ twice reveals

$$
\rho\left(h g h^{-1}\right)=\theta\left(h g, h^{-1}\right)^{-1} \theta(h, g)^{-1} \rho(h) \rho(g) \rho\left(h^{-1}\right) .
$$

Then applying it again to $\rho\left(h h^{-1}\right)$ gives $\rho\left(h^{-1}\right)=\theta\left(h^{-1}, h\right) \rho(h)^{-1}$. Substituting this into the above and using the two-cocycle relation $\theta\left(h g, h^{-1}\right) \theta\left(h^{-1}, h\right)=\theta\left(h g h^{-1}, g\right)$ coming from the triple $\left(h g, h^{-1}, h\right)$ gives

$$
\rho\left(h g h^{-1}\right)=\theta\left(h g h^{-1}, h\right) \theta(h, g)^{-1} \rho(h) \rho(g) \rho(h)^{-1} .
$$

Applying trace gives the requisite relation.

We can define a $\theta$-twisted character to be a function on $G$ which satisfies the conjugation relation in Lemma 5, and then you can show that $\theta$-twisted characters classify $\theta$-twisted representations up to equivalence (see Karpilovsky [12]). We will see below that $\theta$-twisted characters can be thought of as sections of a certain line bundle over the loop groupoid $\Lambda G$.

\subsection{Zero-cocycles, locally-constant functions and integration}

To a zero-cocycle on a groupoid we can associate its integral which is just a complex number.

Suppose that $\mathcal{G}$ is a finite groupoid. A zero-cycle $\beta \in Z^{0}(\mathcal{G}, U(1))$ is the same thing as a locally constant function, that is a $U(1)$-valued function which is constant on connected components of $\mathcal{G}$. We can define $\int_{\mathcal{G}} \beta$ the integral of $\beta$ as follows

$$
\int_{\mathcal{G}} \beta:=\sum_{x \in \mathrm{Ob}(\mathcal{G})} \frac{\beta(x)}{|x \rightarrow|},
$$


where $|x \rightarrow|$ denotes the number of morphisms in $\mathcal{G}$ with $x$ as their source. Essentially this amounts to picking a measure on $\mathcal{G}$ and the point about this measure is that it is invariant under pullback via equivalences, ie, if $F: \mathcal{H} \rightarrow \mathcal{G}$ is an equivalence then $\int_{\mathcal{G}} \beta=\int_{\mathcal{H}} F^{*} \beta$. This follows from an alternative expression for the integral:

$$
\int_{\mathcal{G}} \beta=\sum_{[x] \subset \mathrm{Ob}(\mathcal{G})} \frac{\beta(x)}{|\operatorname{Aut}(x)|},
$$

where $x$ runs over one object from each connected component of $\mathcal{G}$. This is immediate as $\beta$ is constant on connected components and $|[x]||\operatorname{Aut}(x)|=|x \rightarrow|$.

\subsection{One-cocycles, circle-bundles and flat sections}

To a one-cocycle on a groupoid we can associate a "flat complex line bundle" and from that its "space of flat sections" which is a complex vector space.

2.2.1 Circle bundles and associated line bundles For a finite groupoid $\mathcal{G}$ a onecocycle $\alpha \in Z^{1}(\mathcal{G}, U(1))$ is the same thing as a functor $\mathcal{G} \rightarrow \overline{U(1)}$, where as usual $\overline{U(1)}$ means the one object groupoid with $U(1)$ as its group of morphisms-the cocycle condition translates into functoriality under composition of morphisms. Such a functor can also be thought of as a "trivialized $U(1)$-bundle with flat connection" over $\mathcal{G}$, with a copy of the standard $U(1)$ sitting over each object of $\mathcal{G}$ and with parallel transport along a morphism given by the cocycle evaluated on that morphism.

As an aside, note that more generally, a " $U(1)$-bundle with flat connection" over $\mathcal{G}$ would be a functor into the category of principal $U(1)$-spaces (also known as $U(1)-$ torsors). This category is equivalent to the one object category $\overline{U(1)}$, reflecting the fact that in this finite setting, all $U(1)$-bundles are trivializable.

Associated to $\alpha$ as above we have the corresponding "trivialized complex-line bundle with flat connection" which we can denote $\alpha_{\mathbb{C}}: \mathcal{G} \rightarrow \overline{\mathbb{C}}$, this has a standard copy of $\mathbb{C}$ over each object and the same parallel transport as the $U(1)$-bundle. A flat section of the trivialized complex line bundle $\alpha_{\mathbb{C}}$ is then defined to be a map $s: \mathrm{Ob}(\mathcal{G}) \rightarrow \mathbb{C}$ such that for any morphism $f: x_{1} \rightarrow x_{2}$ in $\mathcal{G}$ we have $\alpha(f) s\left(x_{1}\right)=s\left(x_{2}\right)$. It is then a simple observation that for each connected component of $\mathcal{G}$ there is either a one-dimensional or zero-dimensional space of flat sections, depending whether or not $\alpha$ sends all automorphism in that component to the identity in $U(1)$. Below we will see a formula for the number of flat sections of $\alpha_{\mathbb{C}}$ in general.

2.2.2 Inner product on the space of sections Suppose that $s_{1}$ is a section of the line bundle $\alpha_{\mathbb{C}}$ over the groupoid $\mathcal{G}$, then this is a function $s_{1}: \mathrm{Ob}(\mathcal{G}) \rightarrow \mathbb{C}$ with certain 
transformation properties. Now the complex conjugate $\bar{s}_{1}: \mathrm{Ob}(\mathcal{G}) \rightarrow \mathbb{C}$ is actually a section of $\left(\alpha^{-1}\right)_{\mathbb{C}}$ so, given another flat section $s_{2}$, the product $\overline{s_{1}} s_{2}: \mathrm{Ob}(\mathcal{G}) \rightarrow \mathbb{C}$ is just a flat section of the trivial line bundle, or in other words, a function constant on connected components. Thus defining an inner product

$$
\left\langle s_{1}, s_{2}\right\rangle:=\int_{\mathcal{G}} \overline{s_{1}} s_{2}
$$

gives something that is invariant under equivalence of groupoids.

2.2.3 Number of flat sections We can now see the formula for the number of flat sections of the flat line bundle $\alpha_{\mathbb{C}}$ corresponding to the one-cocycle $\alpha$. This can be calculated using the transgression of $\alpha$ which is a zero-cocycle on the loop groupoid (see Section 1.3.3).

Theorem 6 If $\alpha \in Z^{1}(\mathcal{G}, U(1))$ then the number of flat sections of the flat line bundle $\alpha_{\mathbb{C}}$ is given by integrating $\tau(\alpha) \in Z^{0}(\Lambda \mathcal{G}, U(1))$, the transgression of $\alpha$, over the loop groupoid $\Lambda \mathcal{G}$, in other words

$$
\operatorname{dim} \Gamma_{\mathcal{G}}\left(\alpha_{\mathbb{C}}\right)=\int_{\Lambda \mathcal{G}} \tau(\alpha) .
$$

Proof It suffices to prove this in the case that $\mathcal{G}$ is connected as the number of sections is clearly additive under disjoint union. Furthermore, as everything is invariant under equivalence of groupoids, it suffices to prove it in the case when $\mathcal{G}$ has just one object, in other words, when $\mathcal{G}=\bar{G}$ for some finite group $G$. In this case $\alpha \in Z^{1}(\bar{G},(U(1))$ is just a group homomorphism $G \rightarrow U(1)$, and the line bundle $\alpha_{\mathbb{C}}$ has a section precisely when $\alpha$ is trivial.

Looking at the right hand side of the above formula, by definition we have

$$
\int_{\Lambda \bar{G}} \tau(\alpha)=\sum_{x \in G} \frac{\alpha(x)}{|G|} .
$$

However, for any $h$ in $G$ we have

$$
\sum_{x} \frac{\alpha(x)}{|G|} \alpha(h)=\sum_{x} \frac{\alpha(x h)}{|G|}=\sum_{x} \frac{\alpha(x)}{|G|} .
$$

So $\sum_{x} \frac{\alpha(x)}{|G|}(\alpha(h)-1)=0$. Thus

$$
\int_{\Lambda \bar{G}} \tau(\alpha)=\left\{\begin{array}{ll}
1 & \alpha \equiv 1 \\
0 & \text { otherwise }
\end{array}\right\}=\operatorname{dim}\left\{\text { sections of } \alpha_{\mathbb{C}}\right\} .
$$


The above has a generalization, as $\alpha_{\mathbb{C}}: \mathcal{G} \rightarrow \overline{\mathbb{C}}$ can be thought of as a one dimensional representation of $\mathcal{G}$. If we let $F: \mathcal{G} \rightarrow \mathcal{V}$ ect be an ordinary representation of the groupoid $\mathcal{G}$ then we also have, $\Gamma_{\mathcal{G}}(F)$ the space of flat section of $F$, which can be thought of as the invariant part of the representation $F$. The character of $F$ is then a locally constant function on the loop groupoid $\Lambda \mathcal{G}$ and the same proof as above gives the following result.

Proposition 7 For $F: \mathcal{G} \rightarrow \mathcal{V}$ ect a representation of a finite groupoid $\mathcal{G}$ the number of flat sections is given by integrating the character $\chi_{F}$ over the loop groupoid:

$$
\operatorname{dim} \Gamma_{\mathcal{G}}(F)=\int_{\Lambda \mathcal{G}} \chi_{F}
$$

\subsection{Two-cocycles, gerbes and twisted representations}

This subsection follows the same pattern as the previous one, but one categorical level higher. Firstly, given $\theta$ a two-cocycle on a finite groupoid $\mathcal{G}$ a $\theta$-twisted representation is interpreted as a section of a bundle of categories associated to the corresponding "gerbe" over $\mathcal{G}$. These sections naturally form a category. Such a twisted representation can also be viewed as a representation of the twisted groupoid algebra ${ }^{\theta} \mathbb{C} \mathcal{G}$, which is seen to be semisimple. After an appropriate notion of inner product on the category of twisted representations is defined, the idea of character is introduced, and that the representations are classified by their character.

2.3.1 Basics If $\mathcal{G}$ is a finite groupoid and $\theta \in Z^{2}(\mathcal{G}, U(1))$ is a two-cocycle, then by analogy with the finite group case we can define a $\theta$-twisted representation of $\mathcal{G}$ to be something like a representation of $\mathcal{G}$-ie, a functor $\mathcal{G} \rightarrow \mathcal{V}$ ect-but which fails the composition rule in a manner controlled by $\theta$. So we define, $\mathrm{F}$, a $\theta$-twisted representation of $\mathcal{G}$ to be something which associates to each object $x$ a vector space $F(x)$ and to each morphism $g: x_{1} \rightarrow x_{2}$ a linear map $F(g): F\left(x_{1}\right) \rightarrow F\left(x_{2}\right)$, such that $F\left(g_{2}\right) F\left(g_{1}\right)=\theta\left(\left[g_{2} \mid g_{1}\right]\right) F\left(g_{2} \circ g_{1}\right)$ whenever $g_{2}$ and $g_{1}$ are composable. I will denote a twisted representation of $\mathcal{G}$ by $F:{ }^{\theta} \mathcal{G} \rightarrow \mathcal{V}$ ect, this is slightly ambiguous given the notation that follows, but this should not cause any confusion.

As in the finite group case, there are several other ways of thinking of twisted representations. Firstly, and philosophically the gerbel way to think of them, is via the "trivialized central extension" ${ }^{\theta} \mathcal{G}$ defined as follows: objects of ${ }^{\theta} \mathcal{G}$ are the same as objects of $\mathcal{G}$, but the morphism sets are $\operatorname{Mor}_{\theta_{\mathcal{G}}}(x, y):=U(1) \times \operatorname{Mor}_{\mathcal{G}}(x, y)$ with the twisted composition given by $\left(z_{2}, g_{2}\right) \circ\left(z_{1}, g_{1}\right):=\left(\theta\left(\left[g_{2} \mid g_{1}\right]\right) z_{2} z_{1}, g_{2} \circ g_{1}\right)$. This is can be thought of as a central extension written as $\overline{U(1)} \rightarrow{ }^{\theta} \mathcal{G} \rightarrow \mathcal{G}$, which is what I will mean by a gerbe (with flat connection) over $\mathcal{G}$-this point of view is due to 
Moerdijk (see Lupercio and Uribe [14]). A $\theta$-twisted representation of $\mathcal{G}$ is then a representation of $\mathcal{G}$ in which the central $U(1)$ acts in its natural way. An even more appropriate way to think is via the associated "two-vector bundle" $\mathcal{V}$ ect $\rightarrow{ }^{\theta} \mathcal{G}$ Vect $\rightarrow \mathcal{G}$ where we define ${ }^{\theta} \mathcal{G}_{\mathcal{V} \text { ect }}:=\mathcal{V}$ ect $\times \overline{U(1)}^{\theta} \mathcal{G}$, so that

$$
\begin{aligned}
\operatorname{Ob}\left({ }^{\theta} \mathcal{G}_{\mathcal{V} \text { ect }}\right) & :=\operatorname{Ob}(\mathcal{V e c t}) \times \operatorname{Ob}(\mathcal{G}), \\
\operatorname{Mor}_{\mathcal{G}_{\mathcal{V} \text { ect }}}\left(\left(V_{1}, x_{1}\right),\left(V_{2}, x_{2}\right)\right) & :=\operatorname{Mor}_{\mathcal{V}_{\text {ect }}\left(V_{1}, V_{2}\right) \times \operatorname{Mor}_{\mathcal{G}}\left(x_{1}, x_{2}\right),}, \\
\left(\varphi_{2}, g_{2}\right) \circ\left(\varphi_{1}, g_{1}\right) & :=\left(\theta\left(\left[g_{2} \mid g_{1}\right]\right) \varphi_{2} \circ \varphi_{1}, g_{2} \circ g_{1}\right) .
\end{aligned}
$$

A $\theta$-twisted representation of $\mathcal{G}$ is then just a section of ${ }^{\theta} \mathcal{G}$ Vect $\stackrel{\pi}{\longrightarrow} \mathcal{G}$, in other words a functor $\mathcal{G} \stackrel{F}{\longrightarrow} \theta_{\mathcal{G}} \mathcal{V}_{\text {ect }}$ such that $\pi \circ F=\operatorname{Id}_{\mathcal{G}}$.

The collection of $\theta$-twisted representations of $\mathcal{G}$ form a category which I will denote by $\operatorname{Rep}^{\theta}(\mathcal{G})$. The morphisms are the intertwiners, so if $F, F^{\prime}:{ }^{\theta} \mathcal{G} \rightarrow \mathcal{V}$ ect are representations then a morphism $T: F \Rightarrow F^{\prime}$ is essentially a natural transformation, so for each $x \in \mathrm{Ob}(\mathcal{G})$ there is a linear map $T(x): F(x) \rightarrow F^{\prime}(x)$ such that for any $g: x \rightarrow y$ we have $T(y) F(g)=F(g) T(x)$.

Another way to think of a $\theta$-twisted representation of $\mathcal{G}$, which is relevant for the twisted Drinfeld double, is as an ordinary representation of ${ }^{\theta} \mathbb{C G}$ the twisted groupoid algebra, this is defined to be the algebra spanned by the morphisms in $\mathcal{G}$, such that the product $\left\langle g_{2}\right\rangle\left\langle g_{1}\right\rangle$ is zero if $g_{2}$ and $g_{1}$ are not composable in $\mathcal{G}$ and is $\theta\left(\left[g_{2} \mid g_{1}\right]\right)\left\langle g_{2} \circ g_{1}\right\rangle$ otherwise. Ordinary representations of this algebra are then essentially the same thing as $\theta$-twisted representations of the original groupoid, as is explained in the next proposition.

Proposition 8 If $\mathcal{G}$ is a finite groupoid and $\theta \in Z^{2}(\mathcal{G}, U(1))$ is a two-cocycle then the category of representations of the twisted groupoid algebra ${ }^{\theta} \mathbb{C G}$ is equivalent to the category $\operatorname{Rep}^{\theta}(\mathcal{G})$ of $\theta$-twisted representations of the groupoid $\mathcal{G}$.

Proof Suppose that $F:{ }^{\theta} \mathcal{G} \rightarrow \mathcal{V}$ ect is a $\theta$-twisted representation of $\mathcal{G}$, then define the vector space $V:=\bigoplus_{x \in \mathrm{Ob}(\mathcal{G})} F(x)$ and the map $\rho:{ }^{\theta} \mathbb{C G} \rightarrow \operatorname{End}(V)$ when $g: x_{0} \rightarrow x_{1}$ is a morphism in $\mathcal{G}$ by $\rho(\langle g\rangle) v:=F(g) v$ if $v \in F\left(x_{0}\right)$ and zero if $v$ is in any other homogeneous component.

Conversely, suppose that $\rho:{ }^{\theta} \mathbb{C G} \rightarrow \operatorname{End}(V)$ is a representation of the groupoid algebra. To create a $\theta$-twisted representation of the groupoid $\mathcal{G}$ we have to break the vector space $V$ into pieces: for $x \in O b(\mathcal{G})$ define the vector space $F(x):=\rho\left(\left\langle\operatorname{Id}_{x}\right\rangle\right) V$. The fact that the identity in the twisted groupoid algebra splits into a sum of the individual identities in the groupoid which are indecomposable idempotents gives that $V$ is just the direct sum $\bigoplus_{x \in \mathrm{Ob}(\mathcal{G})} F(x)$. The functor $F:{ }^{\theta} \mathcal{G} \rightarrow \mathcal{V}$ ect is then completed 
by defining it on morphisms as follows, if $g: x_{0} \rightarrow x_{1}$ is a morphism in $\mathcal{G}$ then $F(g):=\left.\rho(\langle g\rangle)\right|_{F\left(x_{0}\right)}$.

As in the case of ordinary representations of finite groups, every twisted representation is completely reducible so can be written as a direct sum of irreducible representations.

Theorem 9 If $F^{\prime}$ is a subrepresentation of the $\theta$-twisted representation $F:{ }^{\theta} \mathcal{G} \rightarrow$ Vect, then there is a complementary subrepresentation $F^{\prime \prime}$ so that $F=F^{\prime} \oplus F^{\prime \prime}$.

Proof This is similar to the usual proof for untwisted representations of groups, but takes a little more care.

For each object $x$ in $\mathcal{G}$ pick a projection $P(x): F(x) \rightarrow F(x)$ so that $\operatorname{Im}(P(x))=$ $F^{\prime}(x)$, this means that $P(x)^{2}=P(x)$ and, as $F^{\prime}$ is a subrepresentation, for any morphism $g: x \rightarrow y$ we have $P(y) F(g) P(x)=F(g) P(x)$. Typically, $P$ will not be an intertwiner, but in the usual way we can make it into one. Define

$$
P^{\prime}(x):=\frac{1}{|x \rightarrow|} \sum_{\substack{g \\ x \rightarrow y}} F(g)^{-1} P(y) F(g),
$$

where the sum is over all morphisms coming out of $x$ and $|x \rightarrow|$ is the number of such morphisms. Then it is straight forward (but not without some grittiness) to show that $P^{\prime}(x)^{2}=P^{\prime}(x)$, that $\operatorname{Im}\left(P^{\prime}(x)\right)=F^{\prime}(x)$ and that $P^{\prime}$ is an intertwiner. We can then take the complementary representation $F^{\prime \prime}$ to be ker $P^{\prime}$.

It follows from this that the twisted groupoid algebra ${ }^{\theta} \mathbb{C} \mathcal{G}$ is semisimple.

If we now denote the Grothendieck group of $\operatorname{Rep}^{\theta}(\mathcal{G})$ the category of $\theta$-twisted representations by $\operatorname{Rep}^{\theta}(\mathcal{G})$, then the above means that the representation group $\operatorname{Rep}^{\theta}(\mathcal{G})$ is the free abelian group generated by the equivalence classes of the irreducible representations.

2.3.2 Inner product on representations We can define the functor

$$
\langle-,-\rangle: \mathcal{R e p}^{\theta}(\mathcal{G}) \times \mathcal{R e p}^{\theta}(\mathcal{G}) \rightarrow \mathcal{V e c t} ; \quad\left\langle F_{1}, F_{2}\right\rangle:=\operatorname{Hom}_{\mathcal{R} \operatorname{ep}^{\theta}(\mathcal{G})}\left(F_{1}, F_{2}\right)
$$

which is thought of as an "inner product" on the category of $\theta$-twisted representations. This sort of situation often arises in categories in which there are internal hom functors and duals, this isn't the case in the twisted situation and it is worth explaining this. 
The first thing to do is to understand the contragradient twisted representation. If $F:{ }^{\theta} \mathcal{G} \rightarrow \mathcal{V}$ ect is a $\theta$-twisted representation then define $F^{\vee}$ to be the "functor"

$$
\begin{aligned}
F^{\vee}(x) & :=F(x)^{\vee}=\operatorname{Hom}_{\mathbb{C}}(F(x), \mathbb{C}) ; \\
F^{\vee}\left(g: x_{0} \rightarrow x_{1}\right) & :=\left(\left(F(g)^{-1}\right)^{\vee}: F\left(x_{0}\right)^{\vee} \rightarrow F\left(x_{1}\right)^{\vee}\right) .
\end{aligned}
$$

Note that in general $F(g)^{-1}$ is not the same as $F\left(g^{-1}\right)$, but they differ by a factor of $\theta\left(g, g^{-1}\right)$. A two-line calculation gives that $F^{\vee}$ is a $\theta^{-1}$-twisted representation of $\mathcal{G}$, whereas a one-line calculation gives that the tensor product of a $\theta$-twisted representation and a $\psi$-twisted representation is a $\theta \psi$-twisted representation, so if $F_{1}$ and $F_{2}$ are both $\theta$-twisted representations then $F_{1}^{\vee} \otimes F_{2}$ is a genuine representation of $\mathcal{G}$, in other words a genuine functor $\mathcal{G} \rightarrow \mathcal{V}$ ect, so we can take the space of flat sections

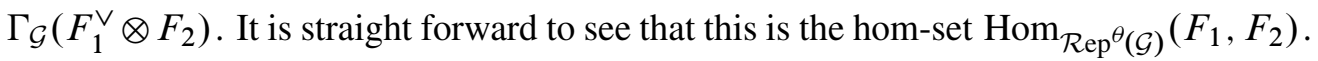

2.3.3 Characters of twisted representations of groupoids We can now essentially copy the construction from twisted representations of finite groups, and classify, up to equivalence, $\theta$-twisted representations of a finite groupoid $\mathcal{G}$ by $\theta$-twisted characters, which are flat sections of a line bundle over the loop groupoid $\Lambda \mathcal{G}$, the line bundle being $\tau(\theta)_{\mathbb{C}}$, the line bundle associated to the circle-bundle transgressed from $\theta$.

Define the character map

$$
\chi: \operatorname{Rep}^{\theta}(\mathcal{G}) \rightarrow \Gamma_{\Lambda \mathcal{G}}\left(\tau(\theta)_{\mathbb{C}}\right) ; \quad F \mapsto \chi_{F} ; \quad \chi_{F}(\gamma):=\operatorname{Tr} \circ F(\gamma) .
$$

The fact that $\chi_{F}$ is indeed a section of $\tau(\theta)_{\mathbb{C}}$, ie, that

$$
\chi_{F}\left(h \gamma h^{-1}\right)=\tau(\theta)([h] \gamma) \chi_{F}(\gamma),
$$

is proved exactly as Lemma 5 . We can call the space of flat sections $\Gamma_{\Lambda \mathcal{G}}\left(\tau(\theta)_{\mathbb{C}}\right)$ the space of $\theta$-twisted characters of $\mathcal{G}$. The fact that the twisted character map $\chi$ is an injection follows from the following result.

Proposition 10 The twisted character map respects the inner products: if $F_{1}$ and $F_{2}$ are $\theta$-twisted representations of $\mathcal{G}$ then

$$
\left\langle\chi_{F_{1}}, \chi_{F_{2}}\right\rangle=\operatorname{dim}\left\langle F_{1}, F_{2}\right\rangle .
$$

Proof There are two preliminary results needed here. The first is that if $F$ and $F^{\prime}$ are respectively $\theta$-twisted and $\phi$-twisted representations of $\mathcal{G}$ then $\chi_{F} \otimes F^{\prime}=\chi_{F} \cdot \chi_{F^{\prime}}$ this follows immediately from the properties of traces.

The second result needed is slightly more awkward to prove but it is that the character of a contragradient representation is the conjugate of the character of the original 
representation: $\chi_{F^{\vee}}=\overline{\chi_{F}}$. Note firstly that the trace of the adjoint of a linear map is equal to the trace of the original map and if $A$ is a linear map with all eigenvalues in $U(1)$ then $\operatorname{Tr} A^{-1}=\overline{\operatorname{Tr} A}$. So for $g$ any automorphism in $\mathcal{G}$ we have $\chi_{F^{\vee}}(g)=$ $\operatorname{Tr}\left(\left(F(g)^{-1}\right)^{\vee}\right)=\operatorname{Tr}\left(F(g)^{-1}\right)$, thus if $F(g)$ has all its eigenvalues in $U(1)$ then this equals $\overline{\operatorname{Tr}(F(g))}=\overline{\chi_{F}(g)}$, and we are done. Hence it suffices to show that $F(g)$ has all its eigenvalues in $U(1)$. Begin by supposing that the eigenvalues of $F(g)$ are $d_{1}, \ldots, d_{m}$, and that $g$ has order $q$ in $G$. The $F(g)^{q}$ differs from $F\left(g^{q}\right)=F(e)=$ Id by a scalar which is a product of factors coming from $\theta$, and as $\theta$ is $U(1)$-valued, this scalar lives in $U(1)$. Thus the eigenvalues of $F(g)^{q}$, ie, $d_{1}^{q}, \ldots, d_{m}^{q}$, all live in $U(1)$ and hence the eigenvalues of $F(g)$ live in $U(1)$.

We can now proceed with the proof of the proposition. Using the above two results together with the ideas of Section 2.3.2 and Proposition 7 we find

$$
\begin{aligned}
\operatorname{dim}\left\langle F_{1}, F_{2}\right\rangle & =\operatorname{dim} \Gamma_{\mathcal{G}}\left(F_{1}^{\vee} \otimes F_{2}\right)=\int_{\Lambda \mathcal{G}} \chi_{F_{1}^{\vee} \otimes F_{2}}=\int_{\Lambda \mathcal{G}} \chi_{F_{1}^{\vee}} \cdot \chi_{F_{2}} \\
& =\int_{\Lambda \mathcal{G}} \overline{\chi F_{1}} \cdot \chi_{F_{2}}=\left\langle\chi_{F_{1}}, \chi_{F_{2}}\right\rangle .
\end{aligned}
$$

From this it is clear that $\theta$-twisted representations are classified up to equivalence by their character, as the set of (equivalence classes of) irreducible twisted representations is mapped bijectively to an orthonormal set. Even better is that this is a full embedding so that the irreducible twisted representations are actually mapped to an orthonormal basis.

Theorem 11 For $\mathcal{G}$ a finite groupoid and $\theta \in Z^{2}(\mathcal{G}, U(1))$, the character map defined above induces an isomorphism of inner product spaces:

$$
\chi: \operatorname{Rep}^{\theta}(\mathcal{G}) \otimes \mathbb{C} \stackrel{\simeq}{\longrightarrow} \Gamma_{\Lambda \mathcal{G}}\left(\tau(\theta)_{\mathbb{C}}\right) .
$$

Proof This follows from Theorem 18 below-which decomposes $\operatorname{Rep}^{\theta}(\mathcal{G})$ as the representation groups of the automorphism groups of the components of $\mathcal{G}$-together with the fact that the theorem holds for finite groups [12].

Applying Theorem 6 to get the dimension of the above spaces gives a formula for the rank of the twisted representation group as follows.

Corollary 12 For $\mathcal{G}$ a finite groupoid, and $\theta \in Z^{2}(\mathcal{G}, U(1))$, $\#\{$ irreducible $\theta$-twisted representations of $\mathcal{G}\}=\int_{\Lambda^{2} \mathcal{G}} \tau^{2}(\theta)$. 
Moreover, specializing to the case when $\mathcal{G}$ is just a group, we can unpack the definition of the right-hand side to obtain:

Corollary 13 For $G$ a finite group and $\theta \in Z_{g p}^{2}(G, U(1))$,

$$
\#\{\text { irreducible } \theta \text {-twisted representations of } G\}=\frac{1}{|G|} \sum_{x g=g x} \frac{\theta(x, g)}{\theta(g, x)} \text {. }
$$

This does not seem to appear in [12] but was undoubtedly known to representation theorists: it is equation (6.40) in the paper of Dijkgraaf and Witten [7].

The above formula can easily be applied to the example in the introduction to this section, to verify that there is one irreducible $\theta_{V}$-twisted representation of $\mathbb{Z}_{2} \times \mathbb{Z}_{2}$, which is of course the two-dimensional representation given.

2.3.4 Dimension In this subsection we look at the "dimension" of the $\theta$-twisted representations of a finite groupoid - that is the space of natural transformations of the identity functor-and show that it is isomorphic to the space of $\tau(\theta)$-twisted class functions on the loop groupoid. This generalizes the fact that the space of natural transformations on the representation category of a finite group is isomorphic to the space of class functions on the group. We begin with a standard theorem:

Theorem 14 If $A$ is a unital algebra and $\operatorname{Rep} A$ is its category of representations, then there is an isomorphism between the centre of $A$ and the natural transformations of the identity functor on $\operatorname{Rep} A$ :

$$
Z(A) \cong \operatorname{Nat}\left(\operatorname{Id}_{\operatorname{Rep} A}, \operatorname{Id}_{\operatorname{Rep} A}\right) .
$$

Proof (Sketch) I will just set up the correspondence and leave the reader to verify the details. Let $A^{\text {reg }}$ denote the regular representation of $A$ on itself, and $e$ denote the identity element of $A$ (considered as an element of $A^{\text {reg }}$ ). Given an element $z$ in the centre of $A$, define the natural transformation of the identity, $\Theta^{z}$, by $\Theta_{(V, \rho)}^{z}(v):=\rho(z) v$. Conversely, given a natural transformation $\Theta$, associate to it the element $\Theta_{A^{\mathrm{rg}}}(e)$ in the centre of $A$.

Now we can identify the centre of a twisted groupoid algebra.

Theorem 15 If a $\mathcal{G}$ is a finite groupoid and $\theta$ is a two-cocycle on $\mathcal{G}$ then the centre of the twisted groupoid algebra ${ }^{\theta} \mathbb{C G}$ is isomorphic to the space of $\tau(\theta)$-twisted class functions on the loop groupoid, ie,

$$
Z\left({ }^{\theta} \mathbb{C} \mathcal{G}\right) \cong \Gamma_{\Lambda \mathcal{G}}\left(\tau(\theta)_{\mathbb{C}}\right)
$$


Proof First we should unpack the right-hand side. An element of $\Gamma_{\Lambda \mathcal{G}}\left(\tau(\theta)_{\mathbb{C}}\right)$ is a function $s: \operatorname{Ob}(\Lambda \mathcal{G}) \rightarrow \mathbb{C}$ such that $s\left(h g h^{-1}\right)=\theta\left(h g h^{-1}, h\right) \theta(h, g)^{-1} s(g)$ for any morphism $h$. Now suppose that $\sum_{g} \lambda_{g}\langle g\rangle$ is in the centre of the twisted group algebra. Then note that $\lambda_{g}=0$ unless $g \in \operatorname{Hom}(x, x)$ for some $x$, because we have for fixed $x$,

$$
\langle g\rangle\left\langle e_{x}\right\rangle=\left\{\begin{array}{ll}
\langle g\rangle & \text { if } \operatorname{source}(g)=x \\
0 & \text { otherwise }
\end{array} \quad \text { and }\left\langle e_{x}\right\rangle\langle g\rangle= \begin{cases}\langle g\rangle & \text { if } \operatorname{target}(g)=x \\
0 & \text { otherwise }\end{cases}\right.
$$

So

$$
\sum_{\text {source }(g)=x} \lambda_{g}\langle g\rangle=\left(\sum_{g} \lambda_{g}\langle g\rangle\right)\left\langle e_{x}\right\rangle=\left\langle e_{x}\right\rangle\left(\sum_{g} \lambda_{g}\langle g\rangle\right)=\sum_{\operatorname{target}(g)=x} \lambda_{g}\langle g\rangle .
$$

Now

$$
\begin{aligned}
\langle h\rangle \sum_{g} \lambda_{g}\langle g\rangle & =\sum_{g} \lambda_{g}\langle g\rangle\langle h\rangle=\sum_{g} \lambda_{g} \theta(g, h)\langle g h\rangle \\
& =\sum_{k} \lambda_{h k h^{-1}} \theta\left(h k h^{-1}, h\right)\langle h k\rangle \\
& =\langle h\rangle \sum_{k} \lambda_{h k h^{-1}} \theta\left(h k h^{-1}, h\right) \theta(h, k)^{-1}\langle k\rangle \\
& =\langle h\rangle \sum_{g} \lambda_{h g h^{-1}} \theta\left(h g h^{-1}, h\right) \theta(h, g)^{-1}\langle g\rangle .
\end{aligned}
$$

Thus multiplying through by $\theta\left(h^{-1}, h\right)^{-1}\left\langle h^{-1}\right\rangle$ to remove the $\langle h\rangle$ at the front, and comparing coefficients gives $\lambda_{h g h^{-1}}=\theta\left(h g h^{-1}, h\right) \theta(h, g)^{-1} \lambda_{g}$. Thus if we define $s(g):=\overline{\lambda_{g}}$, then we obtain a $\tau(\theta)$-twisted class function on $\mathcal{G}$. Conversely, given such an $s$, then the element $\sum_{g \in \mathrm{Ob}(\Lambda \mathcal{G})} \overline{s(g)}\langle g\rangle$ is central in the twisted groupoid algebra.

Putting these two together we obtain the requisite theorem:

Theorem 16 For $\mathcal{G}$ a finite groupoid and $\theta$ a two-cocycle the space of natural transformations of the identity functor on the twisted representation category is isomorphic to the space of $\theta$-twisted class functions on $\mathcal{G}$ :

$$
\operatorname{Nat}\left(\operatorname{Id}_{\operatorname{Rep} A}, \operatorname{Id}_{\operatorname{Rep} A}\right) \cong \Gamma_{\Lambda \mathcal{G}}\left(\tau(\theta)_{\mathbb{C}}\right) .
$$

\subsection{Classifying groupoid representations via group representations}

From a groupoid point of view it is essentially a triviality that a representation of a groupoid is determined by its restriction to the automorphism groups, this is true in the twisted case as well, but one has to be more careful there. 
2.4.1 Construction We will restrict for the moment to the case of connected finite groupoids. Suppose that $x$ is an object of $\mathcal{G}$ and that $\theta \in Z^{2}(\mathcal{G}, U(1))$ is a two-cocycle then there is a restriction cocycle $\left.\theta\right|_{x} \in Z^{2}(\operatorname{Aut}(x), U(1))$. If

$$
\rho:{ }^{\left.\theta\right|_{x}} \operatorname{Aut}(x) \rightarrow \operatorname{Aut}(V)
$$

is a $\left.\theta\right|_{x}$-twisted representation then we can define a $\theta$-twisted representation of $\mathcal{G}$ in the following way. First pick a map $f_{y}: y \rightarrow x$ for every object $y$, with $f_{x}: x \rightarrow x$ being the identity. Now define

for every object $y$ in $\mathcal{G}: \quad F_{\rho}(y):=V$;

$$
\text { for every } g: y \rightarrow z: \quad F_{\rho}(g):=\frac{\theta\left(\left[f_{z} \mid g\right]\right)}{\theta\left(\left[f_{z} g f_{y}^{-1} \mid f_{y}\right]\right)} \rho\left(f_{z} \circ g \circ f_{y}^{-1}\right) \text {. }
$$

The fact that this satisfies $F_{\rho}(h) F_{\rho}(g)=\theta([h \mid g]) F_{\rho}(h \circ g)$ is a slightly brutal but straight forward calculation, this becomes easier after the factor out-front has been explained in Section 2.4.2 below.

Lemma 17 Suppose $\mathcal{G}$ is a finite, connected groupoid, $\theta \in Z^{2}(\mathcal{G}, U(1))$ is a two-cycle, and $x$ is an object of $\mathcal{G}$. Any $\theta$-twisted representation $F:{ }^{\theta} \mathcal{G} \rightarrow \mathcal{V}$ ect is determined by its restriction $\left.F\right|_{x}:{ }^{\left.\theta\right|_{x}} \operatorname{Aut}(x) \rightarrow \operatorname{Aut}(F(x))$ in the sense that $F$ is isomorphic to the representation induced up from $\left.F\right|_{x}$ (for any choice of morphisms $\left\{f_{y}: y \rightarrow x\right\}_{y \neq x}$ ).

Proof We just need to construct a natural isomorphism $T: F \rightarrow F_{\rho}$, for this we take $T(y):=F\left(f_{y}\right)$ then the fact that $T(z) F(g)=F_{\rho}(g) T(y)$ follows when you observe that $F\left(f_{y}\right)^{-1}=\theta\left(\left[f_{y} \mid f_{y}^{-1}\right]\right)^{-1} F\left(f_{y}^{-1}\right)$ and $\theta\left(\left[f_{y} \mid f_{y}^{-1}\right]\right)^{-1} \theta\left(\left[f_{z} g \mid f_{y}^{-1}\right]\right)=$ $\theta\left(\left[f_{z} g f_{y}^{-1} \mid f_{y}\right]\right)^{-1}$.

The case of a nonconnected groupoid is then an immediate corollary:

Theorem 18 The twisted representation group of a finite groupoid splits as the direct sum of the twisted representation groups of its automorphism groups: in other words

$$
\operatorname{Rep}^{\theta}(\mathcal{G}) \cong \bigoplus_{[x] \subset \mathcal{G}} \operatorname{Rep}^{\theta \mid x}(\operatorname{Aut}(x))
$$

where $x$ runs over one object from each connected component of $\mathcal{G}$. 
2.4.2 Explanation As explained in Section 1.2.2 the system of maps $\left\{f_{y}: y \rightarrow x\right\}$ defines a "retraction" $r: \mathcal{G} \rightarrow \overline{\operatorname{Aut}(x)}$ which gives an inverse equivalence to the inclusion $i: \overline{\operatorname{Aut}(x)} \rightarrow \mathcal{G}$. The functor $r \circ i$ is the identity, but the functor $i \circ r$ is only isomorphic to the identity with a natural transformation $T: \operatorname{Id}_{\mathcal{G}} \Rightarrow i \circ r$ being given by $T(y):=f_{y}$.

Given a genuine representation of $\rho: \operatorname{Aut}(x) \rightarrow \operatorname{Aut}(V)$ we can pull this back to $\mathcal{G}$ to get $r^{*} \rho: \mathcal{G} \rightarrow \mathcal{V}$ ect a representation of $\mathcal{G}$. If $\rho$ is the restriction $\left.F\right|_{x}=i^{*} F$ then the representation $r^{*} \circ i^{*} F$ is isomorphic to the original representation via $T^{*} F$.

Unfortunately this argument does not work in the twisted case for the following reason. If $\psi$ is a two-cocycle on $\operatorname{Aut}(x)$ and $\rho:{ }^{\psi} \operatorname{Aut}(x) \rightarrow \operatorname{Aut}(V)$ is a $\psi$-twisted representation then $r^{*} \rho$ is an $r^{*} \psi$-twisted representation, so if $\psi=i^{*} \theta=\left.\theta\right|_{x}$ then $r^{*} \rho$ is an $(i \circ r)^{*} \theta$-twisted representation of $\mathcal{G}$. However, in general $(i \circ r)^{*} \theta$ is not equal to $\theta$ but only cohomologous to it, in other words they will differ by the boundary of a one-cochain $\epsilon$, so $(i \circ r)^{*} \theta=d \epsilon \cdot \theta$ (remember we write it multiplicatively as the cochains take values in $U(1)$ ). Fortunately there is a way around this. Using standard topological methods we can write down a suitable $\epsilon$ by using the natural transformation $T$. It is probably easiest to do it in slightly more generality.

Theorem 19 Suppose $\mathcal{G}$ and $\mathcal{H}$ are groupoids, $K, \check{K}: \mathcal{G} \rightarrow \mathcal{H}$ are isomorphic functors, $T: K \Rightarrow \check{K}$ is any natural transformation, $\theta \in Z^{2}(\mathcal{H}, U(1))$ is a two-cocycle on $\mathcal{H}$, and $\epsilon \in C^{1}(\mathcal{G}, U(1))$ is the one-cochain on $\mathcal{G}$ defined by

$$
\epsilon\left(x_{0} \stackrel{g}{\rightarrow} x_{1}\right):=\frac{\theta\left(\left[T\left(x_{1}\right) \mid K(g)\right]\right)}{\theta\left(\left[\check{K}(g) \mid T\left(x_{0}\right)\right]\right)} .
$$

Then the pullbacks of $\theta$ along $K$ and $\check{K}$ are cohomologous via $\epsilon$, ie,

$$
K^{*}(\theta)=d \epsilon \cdot \check{K}^{*}(\theta) \in Z^{2}(\mathcal{G}, U(1)) .
$$

Furthermore, if $F:{ }^{\theta} \mathcal{H} \rightarrow \mathcal{V}$ ect is a $\theta$-twisted representation then $\epsilon \cdot \check{K}^{*}(F)$ is a $K^{*}(\theta)$ twisted representation which is isomorphic to $K^{*}(F)$ via the natural transformation $F \circ T: K^{*}(F) \Rightarrow \epsilon \cdot \check{K}^{*}(F)$.

Proof Consider the homotopy $H_{T}: \mathcal{B G} \times I \rightarrow \mathcal{B H}$ given in Section 1.2.2. In particular fix a two-simplex $\sigma$ in $\mathcal{B G}$ and look at the prism $P:=H_{T}(\sigma \times I)$ in $\mathcal{B H}$ pictured in Figure 5.

Observe that the boundary of the prism is given by $\partial P=K(\sigma)-\check{K}(\sigma)+H_{T}(\partial \sigma \times I)$. As $\theta$ is a two-cocycle, it is trivial on boundaries such as $\partial P$, thus

$$
\theta(K(\sigma))^{-1} \theta(\check{K}(\sigma)) \theta\left(H_{T}(\partial \sigma \times I)\right)=1,
$$




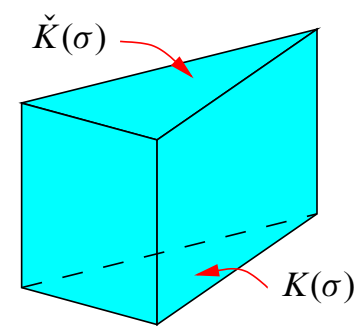

Figure 5: The prism $P$

or in other words,

$$
K^{*} \theta(\sigma)=d \epsilon(\sigma) \cdot \check{K}^{*} \theta(\sigma)
$$

where $\epsilon$ is the one-cochain defined by $\epsilon(\widehat{\sigma}):=\theta\left(H_{T}(\hat{\sigma} \times I)\right)$ for any one-chain $\hat{\sigma}$ on $\mathcal{G}$-remember $d \epsilon(\sigma)$ is, by definition, $\epsilon(\partial \sigma)$. Then we see immediately from the formula given in Section 1.2.2 that for a morphism $g: x_{0} \rightarrow x_{1}$ that $H_{T}([g] \times I)=$ $\left[T\left(x_{1}\right) \mid K(g)\right]-\left[\check{K}(g) \mid T\left(x_{0}\right)\right]$, from which the formula for $\epsilon$ follows.

In the case that we are interested above, we have $\mathcal{H}:=\mathcal{G}, K:=\operatorname{Id}_{\mathcal{G}}, \check{K}:=i \circ r$, so $\check{K}(y \stackrel{g}{\rightarrow} z):=f_{z} \circ g \circ f_{y}^{-1}, T(y):=f_{y}$, from which we get $\check{K}^{*}(F)(y \stackrel{g}{\rightarrow} z)=$ $\left.F\right|_{x}\left(f_{z} \circ g \circ f_{y}^{-1}\right)$, and so we recover the formula from the previous subsection.

\section{The Drinfeld double of a finite group}

We can now see how the general theory of the previous section easily recovers many facts about the twisted Drinfeld double of a finite group. Indeed it is only through this point of view that I understand the Drinfeld double. Firstly we identify the twisted Drinfeld double as the twisted groupoid algebra of the loop groupoid of the finite group. As the loop groupoid of a finite group is the same as the conjugation action groupoid, in Section 3.2 we immediately recover that the representation group of the Drinfeld double is the twisted equivariant $\mathrm{K}$-theory of the group. The fact that the twisted representation group of a groupoid is the direct sum of the representation groups of its automorphism groups translates in Section 3.3 into the decomposition of the representation group of the Drinfeld double à la Dijkgraaf-Pasquier-Roche induction. Finally in Section 3.4 we see how the characters of the Drinfeld double classify representations and are "twisted elliptic characters" on the group.

\subsection{Identifying the twisted Drinfeld double}

For a finite group $G$ and a three-cocycle $\omega \in Z_{\mathrm{gp}}^{3}(G, U(1))$ Dijkgraaf, Pasquier and Roche [6] defined a quasi-Hopf algebra $D^{\omega}(G)$, the twisted Drinfeld double of $G$. 
Freed [8] identified the untwisted Drinfeld double as the groupoid algebra of the action groupoid $\mathcal{G}_{G}\left(G^{\mathrm{c}}\right)$. I am ignoring the coproduct, so we will just be considering $D^{\omega}(G)$ as an associative algebra. Personally, I would take the following theorem to be the definition of $D^{\omega}(G)$ !

Theorem 20 Given a finite group $G$ and a three-cocycle $\omega \in Z_{g p}^{3}(G, U(1))$, let $\tau: Z_{g p}^{3}(G, U(1)) \rightarrow Z^{2}(\Lambda \bar{G}, U(1))$ be the loop transgression map, then the twisted Drinfeld double $D^{\omega}(G)$ of $G$ is isomorphic to the twisted groupoid algebra ${ }^{\tau(\omega)} \mathbb{C}(\Lambda \bar{G})$.

Proof This is just unpacking the definition of the twisted groupoid algebra. A basis of this algebra is indexed by the set of morphisms in $\Lambda \bar{G}$, so we will write the basis element correspond to $g x g^{-1} \stackrel{g}{\longleftarrow} x$ for $x, g \in G$ as $\langle\stackrel{g}{\longleftarrow} x\rangle$. By definition the product of two basis elements is given by

$$
\begin{aligned}
& \langle\stackrel{h}{\leftarrow} y\rangle \cdot\langle\stackrel{g}{\leftarrow} x\rangle:=\delta_{y, g x g^{-1}} \tau(\omega)\left(\left[\stackrel{g}{\leftarrow} g x g^{-1} \mid \stackrel{g}{\leftarrow} x\right]\right)\langle\stackrel{h g}{\longleftarrow} x\rangle, \\
& \tau(\omega)\left(\left[\stackrel{h}{\leftarrow} g x g^{-1} \mid \stackrel{g}{\leftarrow} x\right]\right):=\frac{\omega(h, g, x) \omega\left(h g x(h g)^{-1}, h, g\right)}{\omega\left(h, g x g^{-1}, g\right)} .
\end{aligned}
$$

where

What we have here "up to some changes in notation" [8] is the definition of Dijkgraaf, Pasquier and Roche; actually the notation is essentially the same as that of Freed [8].

This identification has various consequences.

\subsection{Twisted K-theory}

The first consequence of the above theorem can be viewed as a baby version of the Freed-Hopkins-Teleman theorem connecting the Verlinde algebra to ${ }^{\tau(\omega)} K_{G}\left(G^{\mathrm{c}}\right)$ the twisted equivariant K-theory of $G$. The key here is to understand what the definition of the twisted K-group is.

The untwisted equivariant K-group $K_{G}\left(G^{\mathrm{c}}\right)$ of $G$ is defined to be the Grothendieck group of equivariant vector bundles over $G^{\mathrm{c}}$, which is $G$ equipped with the conjugation action. However, an equivariant bundle over $G^{\mathrm{c}}$ is precisely the same thing as a representation of the action groupoid $\mathcal{G}_{G}\left(G^{\mathrm{c}}\right)$; hence $K_{G}\left(G^{\mathrm{c}}\right) \cong \operatorname{Rep}\left(\mathcal{G}_{G}\left(G^{\mathrm{c}}\right)\right)$. So by the twisted equivariant K-theory ${ }^{\theta} K_{G}\left(G^{\mathrm{c}}\right)$ we mean the twisted representation group $\operatorname{Rep}^{\theta}\left(\mathcal{G}_{G}\left(G^{\mathrm{c}}\right)\right)$ for $\theta$ some two-cocycle on the action groupoid $\mathcal{G}_{G}\left(G^{\mathrm{c}}\right)$. Now by Section 1.4.1, the latter groupoid can be identified with the loop groupoid $\Lambda \bar{G}$, so a two-cocycle on the conjugation action groupoid is the same thing as a two-cocycle on the loop groupoid. In particular if $\omega \in Z_{\mathrm{gp}}^{3}(G, U(1))$ then $\tau(\omega) \in Z^{2}(\Lambda \bar{G}, U(1))$ so we can define the twisted equivariant K-theory ${ }^{\tau(\omega)} K_{G}\left(G^{\mathrm{c}}\right)$ to be $\operatorname{Rep}^{\tau(\omega)}(\Lambda \bar{G})$. We can then get the following result. 
Proposition 21 The representation group of the twisted Drinfeld double of a finite group $G$ is naturally isomorphic to the twisted equivariant $K$-group of $G$ :

$$
\operatorname{Rep}\left(D^{\omega}(G)\right) \cong{ }^{\tau(\omega)} K_{G}\left(G^{c}\right)
$$

Proof The twisted equivariant K-theory was defined to be $\operatorname{Rep}^{\tau(\omega)}(\Lambda \bar{G})$, but by Proposition 8 this is the representation group of the twisted groupoid algebra ${ }^{\tau(\omega)} \mathbb{C} \Lambda \bar{G}$, and by Theorem 20 this algebra is just the twisted Drinfeld double $D^{\omega}(G)$.

\subsection{Representation theory}

As the twisted Drinfeld double is a twisted groupoid algebra, the representation theory decomposes according to Theorem 18 into the twisted representation groups of the automorphism groups of the groupoid. In the case of the loop groupoid $\Lambda \bar{G}$, an object is an element of $G$ and the automorphism group of an object $x$ is the centralizer $C_{x}$. Thus by Theorem 20, Theorem 18, and Proposition 8 we get following decomposition.

Theorem 22 The representation group of the twisted Drinfeld double of a finite group $G$ decomposes as the twisted representation groups of the centralizers of the conjugacy classes of $G$ :

$$
\operatorname{Rep}\left(D^{\omega}(G)\right) \cong \bigoplus_{[x] \subset G} \operatorname{Rep}^{\left.\tau(\omega)\right|_{x}}\left(C_{x}\right),
$$

where $x$ runs over one representative from each conjugacy class of $G$.

Given a twisted representation of the centralizer of an element in $G$, the way to get a representation of the twisted Drinfeld double is described in Section 2.4.1 and this is immediately seen to be precisely Dijkgraaf-Pasquier-Roche induction [6].

\subsection{Character theory}

The "character" of a representation $\rho: D^{\omega}(G) \rightarrow \operatorname{End}(V)$ of the twisted Drinfeld double is the function $\chi_{\rho}$ on pairs $(g, x)$ defined by

$$
\chi_{\rho}(g, x):=\operatorname{Tr} \circ \rho(\langle\stackrel{g}{\leftarrow} x\rangle)
$$

and this will vanish unless $x=g x g^{-1}$ (or $x g=g x$ if you prefer). By Section 2.3.3 this is the twisted character of the corresponding $\tau(\omega)$-twisted representation of the loop groupoid $\Lambda \bar{G}$, so it will be a section of the line bundle $\tau^{2}(\omega)_{\mathbb{C}}$ over $\Lambda^{2} \bar{G}$. Such 
a section is just a function $\chi$ on pairs $(g, x) \in G \times G$ such that $x g=g x$ (so that $\chi(g, x)$ really means $\chi(x \stackrel{g}{\leftarrow} x))$ such that for any $h \in G$

$$
\begin{aligned}
\chi\left(h g h^{-1}, h x h^{-1}\right) & =\tau^{2}(\omega)([h](x \stackrel{g}{-} x)) \chi(g, x) \\
& =\frac{\tau(\omega)\left(\left[h g h^{-1} \mid h\right] x\right)}{\tau(\omega)([h \mid g] x)} \chi(g, x) \\
& =\frac{\omega(h, x, g) \omega\left(h g h^{-1}, h, x\right) \omega\left(h x h^{-1}, h g h^{-1}, h\right)}{\omega(h, g, x) \omega\left(h x h^{-1}, h, g\right) \omega\left(h g h^{-1}, h x h^{-1}, h\right)} \chi(g, x) .
\end{aligned}
$$

We can call functions of this form $\omega$-twisted elliptic characters of $G$. Thus we obtain a map

$$
\chi: \operatorname{Rep}\left(D^{\omega}(G)\right) \rightarrow\{\omega \text {-twisted elliptic characters of } G\}
$$

Theorem 11 gives that representations of the Drinfeld double are classified by their character:

Theorem 23 The complexification of the character map is an isomorphism

$$
\chi: \operatorname{Rep}\left(D^{\omega}(G)\right) \otimes \mathbb{C} \stackrel{\cong}{\longrightarrow}\{\omega \text {-twisted elliptic characters of } G\}
$$

and an orthonormal basis of the $\omega$-twisted elliptic characters of $G$ is given by the characters of the irreducible representations of $D^{\omega}(G)$.

Using Corollary 12 we can give the dimension of the space of $\omega$-twisted elliptic characters, which is thus the number of irreducible representations of the twisted Drinfeld double.

Theorem 24 The number of irreducible representations of the twisted Drinfeld double $D^{\omega}(G)$ is given by

$$
\operatorname{rank} \operatorname{Rep}\left(D^{\omega}(G)\right)=\int_{\Lambda^{3} \bar{G}} \tau^{3}(\omega)=\frac{1}{|G|} \sum \frac{\omega(h, x, g) \omega(g, h, x) \omega(x, g, h)}{\omega(h, g, x) \omega(x, h, g) \omega(g, x, h)}
$$

where the sum is over triples $(h, g, x) \in G \times G \times G$ which mutually commute.

Actually this is immediately seen to be the invariant of the three-torus, which is decomposed into six three-simplices as in Figure 6.

A simple example is given by taking $G=\mathbb{Z}_{2} \times \mathbb{Z}_{2} \times \mathbb{Z}_{2}$, with generators $a, b$ and $c$ and with three-cocycle $\omega \in Z^{3}\left(\mathbb{Z}_{2} \times \mathbb{Z}_{2} \times \mathbb{Z}_{2}, U(1)\right)$ defined by

$$
\omega\left(a^{u_{a}} b^{u_{b}} c^{u_{c}}, a^{v_{a}} b^{v_{b}} c^{v_{c}}, a^{w_{a}} b^{w_{b}} c^{w_{c}}\right)=(-1)^{u_{a} \cdot v_{b} \cdot w_{c}} .
$$




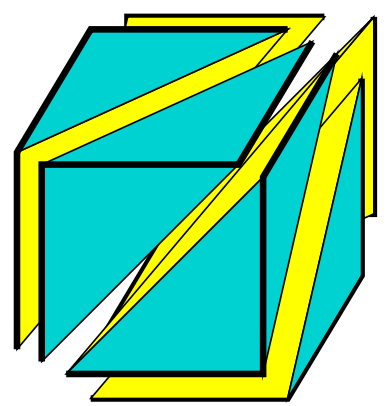

Figure 6: The three-torus decomposed

A quick calculation on maple of the previous formula gives

$$
\operatorname{rank} \operatorname{Rep}\left(D^{\omega}\left(\mathbb{Z}_{2} \times \mathbb{Z}_{2} \times \mathbb{Z}_{2}\right)\right)=22 .
$$

A little more investigation reveals that this is accounted for by 8 one-dimensional representations and 14 two-dimensional representations. A simple check on these numbers is that $\left|\mathbb{Z}_{2} \times \mathbb{Z}_{2} \times \mathbb{Z}_{2}\right|^{2}=64=14 \times 2^{2}+8 \times 1^{2}$.

\section{References}

[1] D Altschüler, A Coste, Quasi-quantum groups, knots, three-manifolds, and topological field theory, Comm. Math. Phys. 150 (1992) 83-107 MR1188498

[2] D Altschüler, A Coste, J-M Maillard, Representation theory of twisted group double, Ann. Fond. Louis de Broglie 29 (2004) 681-694 MR2146291

[3] B H Bartlett, S Willerton, work in progress

[4] D J Benson, Representations and cohomology. II. Cohomology of groups and modules, Cambridge Studies in Advanced Math. 31, Cambridge University Press (1991) MR1156302

[5] J-L Brylinski, D A McLaughlin, The geometry of degree-four characteristic classes and of line bundles on loop spaces. I, Duke Math. J. 75 (1994) 603-638 MR1291698

[6] R Dijkgraaf, V Pasquier, P Roche, Quasi Hopf algebras, group cohomology and orbifold models, Nuclear Phys. B Proc. Suppl. 18B (1990) 60-72 (1991) MR1128130 Recent advances in field theory (Annecy-le-Vieux, 1990)

[7] R Dijkgraaf, E Witten, Topological gauge theories and group cohomology, Comm. Math. Phys. 129 (1990) 393-429 MR1048699

[8] DS Freed, Higher algebraic structures and quantization, Comm. Math. Phys. 159 (1994) 343-398 MR1256993 
[9] D S Freed, F Quinn, Chern-Simons theory with finite gauge group, Comm. Math. Phys. 156 (1993) 435-472 MR1240583

[10] P Gajer, Geometry of Deligne cohomology, Invent. Math. 127 (1997) 155-207 MR1423029

[11] K Gomi, Y Terashima, A fiber integration formula for the smooth Deligne cohomology, Internat. Math. Res. Notices (2000) 699-708 MR1772521

[12] G Karpilovsky, Projective representations of finite groups, Monographs and Textbooks in Pure and Applied Math. 94, Marcel Dekker, New York (1985) MR788161

[13] E Lupercio, B Uribe, Loop groupoids, gerbes, and twisted sectors on orbifolds, from: "Orbifolds in mathematics and physics (Madison, WI, 2001)", Contemp. Math. 310, Amer. Math. Soc. (2002) 163-184 MR1950946

[14] E Lupercio, B Uribe, Gerbes over orbifolds and twisted K-theory, Comm. Math. Phys. 245 (2004) 449-489 MR2045679

[15] E Lupercio, B Uribe, Inertia orbifolds, configuration spaces and the ghost loop space, Q. J. Math. 55 (2004) 185-201 MR2068317

[16] E Lupercio, B Uribe, Holonomy for gerbes over orbifolds, J. Geom. Phys. 56 (2006) 1534-1560 MR2240409

[17] E Lupercio, B Uribe, M A Xicotencatl, Orbifold string topology, Geom. Topol. 12 (2008)

[18] R J Milgram, The bar construction and abelian $H$-spaces, Illinois J. Math. 11 (1967) 242-250 MR0208595

[19] NP Strickland, $K(N)$-local duality for finite groups and groupoids, Topology 39 (2000) 733-772 MR1760427

Department of Pure Mathematics, University of Sheffield Hicks Building, Hounsfield Road, Sheffield, S3 7RH, UK S.Willerton@sheffield.ac.uk

Received: 19 December 2006 